\title{
FLUID CRITICAL BEHAVIOR AT LIQUID-GAS PHASE TRANSITION: ANALYTIC METHOD FOR MICROSCOPIC DESCRIPTION
}

\author{
I.V. PYLYUK \\ Institute for Condensed Matter Physics \\ of the National Academy of Sciences of Ukraine, \\ 1 Svientsitskii Str., 79011 Lviv, Ukraine \\ E-mail: piv@icmp.lviv.ua
}

The behavior of fluids in the vicinity of the liquid-gas critical point is studied within the cell fluid model framework. The analytic method for deriving the equation of state of a cell fluid model in the low-temperature region $\left(T<T_{c}\right)$ is developed using the renormalization group transformation within the collective variables approach. Mathematical description within the grand canonical ensemble is illustrated by an example of the Morse interaction potential possessing the Fourier transform. A specific feature of the proposed method lies in the possibility to use exclusively microscopic characteristics of a fluid (parameters of the interaction potential) for calculating macroscopic quantities (pressure and other thermodynamic quantities) without involving the hard-spheres reference system. The grand partition function, thermodynamic potential, and equation of state of the model near the critical point are derived taking into account the non-Gaussian (quartic) distribution of order parameter fluctuations. A nonlinear equation, which links the density and the chemical potential, is presented and solved. Graphs of the dependence of the density on the chemical potential are plotted for various values of the relative temperature. The numerical estimates of the critical-point parameters for potassium, obtained in addition to the estimates for sodium, are given. The calculated critical-point parameters for liquid alkali metals (sodium and potassium) are in accord with experimental data. The coexistence curve for sodium is plotted and compared with other authors' data in the immediate vicinity of $T_{c}$, where theoretical and experimental researches are difficult to carry out. The differences between the obtained results and the earlier published results for $T>T_{c}$ are discussed.

PACS numbers: 05.70.Ce, 64.60.F-, 64.70.F- 


\section{List of notations}

\begin{tabular}{|c|c|}
\hline Symbol & Meaning \\
\hline$N_{v}$ & number of cells \\
\hline$c$ & linear cell size \\
\hline$v$ & volume of cubic cell \\
\hline$T$ & temperature \\
\hline$T_{c}$ & critical temperature \\
\hline$\Xi$ & grand partition function \\
\hline$W(k)$ & Fourier transform of effective potential \\
\hline$Q_{n}$ & partial partition function of $n$th layer \\
\hline$\Xi_{I G R}$ & $\begin{array}{l}\text { contribution to grand partition function from inverse } \\
\text { Gaussian regime }\end{array}$ \\
\hline$\tilde{h}$ & renormalized external field \\
\hline$h_{c m}$ & temperature field \\
\hline$\tau$ & relative temperature \\
\hline$\Omega$ & thermodynamic potential \\
\hline$\Omega_{\mu}$ & $\begin{array}{l}\text { thermodynamic potential contribution corresponding to } \\
\text { partition function component } G_{\mu}\end{array}$ \\
\hline$\Omega_{r}$ & $\begin{array}{l}\text { thermodynamic potential contribution corresponding to } \\
\text { partition function component }\left(Q\left(r_{0}\right)\right)^{N_{v}}\end{array}$ \\
\hline$\Omega_{C R}^{(-)}$ & $\begin{array}{l}\text { contribution to thermodynamic potential from critical } \\
\text { regime, } T<T_{c}\end{array}$ \\
\hline$\gamma_{01}, \gamma_{02}, \gamma_{03}$ & coefficients for analytic part of $\Omega_{C R}^{(-)}$ \\
\hline$\Omega_{C R}^{(s)^{\prime}}$ & singular part of $\Omega_{C R}^{(-)}$ \\
\hline $\bar{\gamma}^{(-)}$ & coefficient for singular part $\Omega_{C R}^{(s)^{\prime}}, T<T_{c}$ \\
\hline$\Omega_{T R}^{(-)}$ & $\begin{array}{l}\text { contribution to thermodynamic potential from transition } \\
\text { region, } T<T_{c}\end{array}$ \\
\hline$\Omega_{I G R}$ & $\begin{array}{l}\text { contribution to thermodynamic potential from inverse } \\
\text { Gaussian regime }\end{array}$ \\
\hline$\Omega_{a}$ & analytic part of thermodynamic potential \\
\hline$\Omega_{s}^{(-)}$ & nonanalytic part of thermodynamic potential, $T<T_{c}$ \\
\hline$\Omega_{0}^{(-)}$ & $\begin{array}{l}\text { thermodynamic potential part associated with shift of } \\
\text { variable } \rho_{0}, T<T_{c}\end{array}$ \\
\hline $\bar{n}$ & average density \\
\hline$\mu, M$ & chemical potentials \\
\hline$P$ & pressure \\
\hline
\end{tabular}




\section{Introduction}

For more than a century, the behavior of multiparticle systems in the liquid and gas phases attracts the attention of scientists. The task of the theoretical description of the properties of fluids on the level of microscopic interactions between particles remains relevant [1]. Especially significant is the problem of describing fluid in the vicinity of and below the critical temperature $T_{c}$. Below $T_{c}$, two phases can coexist (a gas at low density and a liquid at high density).

The study of the behavior of fluids is often carried out with the use of the concept reference system. The role of the latter is usually played by the system of hard spheres (see, for example, [2 [5]). The novelty of the approach developed in this paper is to use exclusively microscopic characteristics of the model (parameters of the interaction potential) for obtaining macroscopic quantities (pressure and other thermodynamic quantities) without involving the hard-spheres system. The temperature region below the critical temperature $T_{c}$ is considered. The refusal to use the hard-spheres reference system within the collective variables approach [6,7] is due to several reasons. One of them (in the presence of the hard-spheres reference system) is the use of different spaces of variables in calculating the Jacobian of transition to collective variables and grand partition function. If the Jacobian of the transition is calculated in Cartesian space, then the grand partition function of the fluid system is calculated in the space of the collective variables. Methods of calculation in the two spaces mentioned above are fundamentally different, and therefore require the use of different approximations that are difficult to reconcile with each other (to determine common accuracy). The problem of coordinating accuracy in different spaces as well as the problem of reconciling the average number of particles in the reference system and their total number can be avoided in the mathematical description without involving the hard-spheres reference system. In the framework of this approach, the reference system is not introduced from the outside, but appears at a certain stage in the calculation of the grand partition function and is not related to the presence of additional chemical potential. Here one chemical potential for fluid is used.

The theoretical description of the critical behavior of a simple fluid is carried out within the framework of a cell model. The volume of the system $V$ composed of $N$ interacting particles is conventionally divided into $N_{v}$ cells, each of volume $v=V / N_{v}=c^{3}$ ( $c$ is the linear cell size). It should be 
noted that, in contrast to a cell gas model (where it is assumed that a cell may contain only one particle or does not contain any particle) [8, 9], a cell within this approach may contain more than one particle. Instead of the distance between the particles, the distance between the centers of the cells is introduced. The cell interaction potential is chosen in the form of the Morse potential possessing the Fourier transform. Despite the great successes in the investigation of Morse fluids made by means of various methods (for example, the $N p T$ plus test particle method [10], the grand-canonical transition matrix Monte Carlo method [11], the integral equations approach [12], molecular dynamics simulations in a canonical ensemble [13]), the study and statistical description of the behavior of the mentioned fluids near the critical point on the microscopic level without any general assumptions are still of interest.

The present theoretical studies supplement the results of paper [14], in which a method for calculating the equation of state of the fluid system is developed in the case of $T>T_{c}$. Parameters of interaction potential, which are necessary for quantitative estimates, correspond to data for $\mathrm{Na}$ (sodium) and $\mathrm{K}$ (potassiun) [11]. Expressions for the quantities mentioned and not given here can be found in [14]. It should be noted that the temperature region $T<T_{c}$ differs significantly from the region $T>T_{c}$. The reason is that in the system at $T<T_{c}$ the order parameter appears. The influence of this order parameter will be taken into account in the process of determining the system's point of exit from the critical fluctuation regime.

Briefly describe the structure of the present paper. Sec. 2, which follows the introduction (Sec. 1), contains the basic output relations, which are valid for temperatures below $T_{c}$. Here the expression for the grand partition function of the model that it is necessary to calculate for obtaining the thermodynamic potential as well as formulas for the quantities included in this expression are presented. Scheme for calculation of the thermodynamic potential is given in Sec. 3. The basic idea of such a calculation within the approach of collective variables lies in the separate inclusion of contributions from short-wave and long-wave fluctuations of the order parameter (see, for example, [15]). Short-wave fluctuations are characterized by a renormalization group symmetry and are described by a non-Gaussian distribution. They correspond to the region of the critical regime. The region of the inverse Gaussian regime is associated with long-wave fluctuations. The calculation of the contribution to the thermodynamic potential from long-wave fluctuations is based on the use of the Gaussian distribution as the basis one. Here, we have developed a direct method of calculations with the results obtained by 
taking into account the short-wave fluctuations as initial parameters. In the same Sec. 3, the expression for short-wave part of the thermodynamic potential (the contribution from the critical regime of fluctuations) is presented. The procedure for calculating the long-wave part of the thermodynamic potential (the contribution from the inverse Gaussian regime of fluctuations) is developed in Sec. 4. Using the obtained results, the complete expression for the thermodynamic potential is written. A method for constructing the equation of state of a cell fluid model for $T<T_{c}$ is described in Sec. 5, taking into account non-Gaussian fluctuations. The critical-point parameters calculated for $\mathrm{Na}$ and $\mathrm{K}$ as well as the binodal curve obtained for $\mathrm{Na}$ in the immediate vicinity of $T_{c}$ are given. Concise conclusions are presented in Sec. 6. Some supplementary interim results are provided in two appendices. This makes it easier to read the article.

\section{Basic expressions at temperatures below $T_{c}$}

The grand partition function of the cell fluid model can be written in the following form [14,16]:

$$
\Xi=\sum_{N=0}^{\infty} \frac{(z)^{N}}{N !} \int_{V}(d x)^{N} \exp \left[-\frac{\beta}{2} \sum_{\mathbf{l}_{1}, \mathbf{l}_{2} \in \Lambda} \tilde{U}_{l_{12}} \rho_{\mathbf{l}_{1}}(\eta) \rho_{\mathbf{l}_{2}}(\eta)\right] .
$$

Here $z=e^{\beta \mu}$ is the activity, $\beta=1 /(k T)$ is the inverse temperature, and $\mu$ is the chemical potential. Integration with respect to coordinates of all the particles $x_{i}=\left(x_{i}^{(1)}, x_{i}^{(2)}, x_{i}^{(3)}\right)$ is noted as $\int_{V}(d x)^{N}=\int_{V} d x_{1} \cdots \int_{V} d x_{N}$, and $\eta=\left\{x_{1}, \ldots, x_{N}\right\}$ is the set of coordinates. The interaction potential $\tilde{U}_{l_{12}}$ is a function of the distance $l_{12}=\left|\mathbf{l}_{1}-\mathbf{l}_{2}\right|$ between cells. Each vector $\mathbf{l}_{i}$ belongs to the set

$$
\Lambda=\left\{\mathbf{l}=\left(l_{1}, l_{2}, l_{3}\right) \mid l_{i}=c m_{i} ; m_{i}=1,2, \ldots, N_{a} ; i=1,2,3 ; N_{v}=N_{a}^{3}\right\},
$$

where $N_{a}$ is the number of cells along each axis. The occupation numbers of cells

$$
\rho_{\mathbf{l}}(\eta)=\sum_{x \in \eta} I_{\Delta_{\mathbf{l}}(x)}
$$

appearing in Eq. (11) are defined by the characteristic functions (indicators)

$$
I_{\Delta_{\mathrm{l}}(x)}=\left\{\begin{array}{lll}
1, & \text { if } & x \in \Delta_{\mathrm{l}} \\
0, & \text { if } & x \notin \Delta_{\mathrm{l}}
\end{array}\right.
$$


which identify the particles in each cubic cell $\Delta_{\mathrm{l}}=(-c / 2, c / 2]^{3} \subset \mathbb{R}^{3}$ and their contribution to the interaction of a model. In further calculations, the interaction potential $\tilde{U}_{l_{12}}$ will be chosen in the form of the Morse potential:

$$
\tilde{U}_{l_{12}}=\Psi_{l_{12}}-U_{l_{12}} ; \quad \Psi_{l_{12}}=D e^{-2\left(l_{12}-1\right) / \alpha_{R}}, \quad U_{l_{12}}=2 D e^{-\left(l_{12}-1\right) / \alpha_{R}} .
$$

Here $\alpha_{R}=\alpha / R_{0}$, and $\alpha$ is the effective interaction radius. The parameter $R_{0}$ corresponds to the minimum of the function $\tilde{U}_{l_{12}}\left(\tilde{U}\left(l_{12}=1\right)=-D\right.$ determines the depth of potential well). It should be noted that in terms of convenience, the $R_{0^{-}}$-units are used for length measuring. As a result, $R_{0^{-}}$and $R_{0}^{3}$-units are used for the linear size of each cell $c$ and volume $v$, respectively.

In the set of the collective variables $\rho_{\mathbf{k}}$, the general functional representation of the grand partition function of the cell fluid model has the form (see [14, 16])

$$
\begin{aligned}
& \Xi=\int(d \rho)^{N_{v}} \exp \left[\beta \mu \sqrt{N_{v}} \rho_{0}+\frac{\beta}{2} \sum_{\mathbf{k} \in \mathcal{B}} W(k) \rho_{\mathbf{k}} \rho_{-\mathbf{k}}\right] \\
& \times \prod_{l=1}^{N_{v}}\left[\sum_{m=0}^{\infty} \frac{v^{m}}{m !} e^{-p m^{2}} \delta\left(\rho_{\mathbf{l}}-m\right)\right] .
\end{aligned}
$$

As is seen from Eq. (5), the occupation numbers of cells $\rho_{\mathbf{l}}(\eta)$ can take on values $m=0,1,2, \ldots$. Due to the term $e^{-p m^{2}}$, the probability of hosting many particles in a single cell is very small. It should be noted that

$$
(d \rho)^{N_{v}}=\prod_{\mathbf{k} \in \mathcal{B}} d \rho_{\mathbf{k}}
$$

The wave vector $\mathbf{k}$ belongs to the set

$\mathcal{B}=\left\{\mathbf{k}=\left(k_{1}, k_{2}, k_{3}\right) \mid k_{i}=-\frac{\pi}{c}+\frac{2 \pi}{c} \frac{n_{i}}{N_{a}} ; n_{i}=1,2, \ldots, N_{a} ; i=1,2,3 ; N_{v}=N_{a}^{3}\right\}$.

The Brillouin zone $\mathcal{B}$ corresponds to the volume of periodicity $\Lambda$ with cyclic boundary conditions. The parameter $p$ characterizing the reference system is defined as

$$
p=\frac{1}{2} \beta \chi \Psi(0)
$$

and

$$
W(k)=U(k)-\Psi(k)+\chi \Psi(0)
$$


is the Fourier transform of the effective potential of interaction. The Fourier transforms of the attractive and repulsive parts of the Morse potential $\left(U_{l_{12}}\right.$ and $\Psi_{l_{12}}$ in Eqs. (44), respectively) satisfy the expressions

$$
U(k)=U(0)\left(1+\alpha_{R}^{2} k^{2}\right)^{-2}, \quad \Psi(k)=\Psi(0)\left(1+\alpha_{R}^{2} k^{2} / 4\right)^{-2},
$$

where

$$
U(0)=16 D \pi \frac{\alpha_{R}^{3}}{v} e^{R_{0} / \alpha}, \quad \Psi(0)=D \pi \frac{\alpha_{R}^{3}}{v} e^{2 R_{0} / \alpha} .
$$

The positive parameter $\chi$ forms the Jacobian of transition to collective variables.

The expression (5) for the grand partition function of the cell fluid model in the approximation of the simplest non-Gaussian quartic fluctuation distribution (the $\rho^{4}$ model) can be written as [14, 16]

$$
\begin{aligned}
& \Xi=g_{W} e^{N_{v}\left(E_{\mu}-a_{0}\right)} \int(d \rho)^{N_{v}} \exp \left[M N_{v}^{1 / 2} \rho_{0}\right. \\
& -\frac{1}{2} \sum_{\mathbf{k} \in \mathcal{B}} d(k) \rho_{\mathbf{k}} \rho_{-\mathbf{k}} \\
& \left.-\frac{a_{4}}{4 !} N_{v}^{-1} \sum_{\substack{\mathbf{k}_{1}, \ldots, \mathbf{k}_{4} \\
\mathbf{k}_{i} \in \mathcal{B}}} \rho_{\mathbf{k}_{1}} \cdots \rho_{\mathbf{k}_{4}} \delta_{\mathbf{k}_{1}+\cdots+\mathbf{k}_{4}}\right] .
\end{aligned}
$$

Here

$$
\begin{aligned}
& g_{W}=\prod_{\mathbf{k} \in \mathcal{B}}(2 \pi \beta W(k))^{-1 / 2}, \\
& E_{\mu}=-\frac{\beta W(0)}{2}\left(M+\tilde{a}_{1}\right)^{2}+M a_{34}+\frac{1}{2} d(0) a_{34}^{2}-\frac{a_{4}}{24} a_{34}^{4}, \\
& a_{34}=-a_{3} / a_{4}, \\
& M=\mu / W(0)-\tilde{a}_{1}, \quad \tilde{a}_{1}=a_{1}+d(0) a_{34}+\frac{a_{4}}{6} a_{34}^{3}, \\
& d(k)=\frac{1}{\beta W(k)}-\tilde{a}_{2}, \quad \tilde{a}_{2}=\frac{a_{4}}{2} a_{34}^{2}-a_{2},
\end{aligned}
$$

and $\delta_{\mathbf{k}_{1}+\cdots+\mathbf{k}_{4}}$ is the Kronecker symbol. In the case when $R_{0} / \alpha=2.9544$ (which is typical of $\mathrm{Na}[11]), \chi=1.1243(p=1.8100)$, and $v=2.4191$ (see [14,16]), we obtain the following quantities:

$$
\begin{aligned}
& a_{0}=-0.3350, \quad a_{1}=-0.2862, \quad a_{2}=-0.2073 \\
& a_{3}=-0.0938, \quad a_{4}=0.0376, \quad W(0)=17.7687 .
\end{aligned}
$$


We shall proceed from the expression for the grand partition function of the cell fluid model below the critical temperature

$$
\Xi=G_{\mu}\left(Q\left(r_{0}\right)\right)^{N_{v}}\left(\prod_{n=1}^{n_{p}^{\prime}} Q_{n}\right) \Xi_{I G R} .
$$

This result is obtained by step-by-step calculation of partition function within the collective variables approach [by "layer-by-layer" integration in Eq. (9) with respect to collective variables]. The formal part of the procedure has already been presented for $T>T_{c}$ in [14. Here, as in the case of $T>T_{c}$, we have $G_{\mu}=g_{W}(\beta W(0))^{N_{v} / 2} e^{N_{v}\left(E_{\mu}-a_{0}\right)}$. The quantity $Q\left(r_{0}\right)$ appearing in Eq. (11) corresponds to the contribution to the grand partition function from the large values of the wave vector [14], and $Q_{n}$ is the partial partition function of the $n$th layer of the phase space of the collective variables [17]. The upper product limit $n_{p}^{\prime}$ in formula (11) is the number of the layer determining the point of exit of the system from the critical regime of the order parameter fluctuations. For $T<T_{c}$, the quantity $n_{p}^{\prime}$ is defined as [18]

$$
n_{p}^{\prime}=-\frac{\ln \left(\tilde{h}^{2}+h_{c m}^{2}\right)}{2 \ln E_{1}}-1 .
$$

Here the renormalized external field

$$
\tilde{h}=M(\beta W(0))^{1 / 2}
$$

is a function of the chemical potential. The temperature field

$$
h_{c m}=\tilde{\tau}_{1}^{p_{0}}
$$

is characterized by the renormalized relative temperature

$$
\tilde{\tau}_{1}=-\tau \frac{c_{11}}{q} E_{2}^{n_{0}}
$$

and the exponent

$$
p_{0}=\frac{\ln E_{1}}{\ln E_{2}}
$$

where $\tau=\left(T-T_{c}\right) / T_{c}$, and $E_{l}$ are eigenvalues of the renormalization group linear transformation matrix. The quantities $c_{11}$ and $q$ are the same as in 
the case of $T>T_{c}$. It should be noted that in the case of $T>T_{c}$ (see [14]), the temperature field $h_{c}=\tilde{\tau}^{p_{0}}$, where $\tilde{\tau}=\tau c_{11} / q$, was introduced in the expression for the exit point instead of $h_{c m}$. The variable $\tilde{\tau}$ differs from $\tilde{\tau}_{1}$ since the factor $E_{2}^{n_{0}}$ is absent. The quantity $n_{0}=n_{p}-n_{p}^{\prime}$ (at $\tilde{h}=0$ ) characterizing the difference between the points of exit from the critical fluctuation regime at $T>T_{c}\left(n_{p}\right)$ and $T<T_{c}\left(n_{p}^{\prime}\right)$ appears due to a nonzero order parameter for $T<T_{c}$.

The quantity

$$
\Xi_{I G R}=2^{\left(N_{n_{p}^{\prime}+1}-1\right) / 2}\left[Q\left(P_{n_{p}^{\prime}}\right)\right]^{N_{n_{p}^{\prime}+1}} \Xi_{n_{p}^{\prime}+1}
$$

appearing in Eq. (11) determines the contribution to the grand partition function from long-wave fluctuations of the order parameter. Here $N_{n_{p}^{\prime}+1}=$ $N_{v} s^{-3\left(n_{p}^{\prime}+1\right)}, s$ is the parameter of division of the phase space of the collective variables $\rho_{\mathbf{k}}$ into layers. As in the case of $T>T_{c}$, the calculations will be made for the optimal parameter $s=s^{*}=3.5977$ nullifying the average value of the coefficient in the term with the second power of the variable in the effective density of measure at a fixed point. The quantity $Q\left(P_{n}\right)$ is defined in [7, 14], and $\Xi_{n_{p}^{\prime}+1}$ satisfies the expression

$$
\begin{aligned}
& \Xi_{n_{p}^{\prime}+1}=\int(d \rho)^{N_{n_{p}^{\prime}+1}} \exp \left[a_{1}^{\left(n_{p}^{\prime}+1\right)} \sqrt{N_{n_{p}^{\prime}+1}} \rho_{0}\right. \\
& -\frac{1}{2} \sum_{\mathbf{k} \in \mathcal{B}_{n_{p}^{\prime}+1}^{\prime}} g_{n_{p}^{\prime}+1}(k) \rho_{\mathbf{k}} \rho_{-\mathbf{k}} \\
& \left.-\frac{a_{4}^{\left(n_{p}^{\prime}+1\right)}}{4 !} N_{n_{p}^{\prime}+1}^{-1} \sum_{\substack{\mathbf{k}_{\mathbf{1}}, \ldots, \mathbf{k}_{4} \\
\mathbf{k}_{i} \in \mathcal{B}_{n_{p}^{\prime}+1}^{\prime}}} \rho_{\mathbf{k}_{1}} \cdots \rho_{\mathbf{k}_{4}} \delta_{\mathbf{k}_{1}+\cdots+\mathbf{k}_{4}}\right] .
\end{aligned}
$$

The coefficients $a_{1}^{(n)}, g_{n}(0)$, and $a_{4}^{(n)}$ are connected with the coefficients for the $(n+1)$ th layer through the recurrence relations (see [14]), whose solutions are used for calculating the thermodynamic potential of the fluid system. The quantity $b$ appearing in the equality $g_{n_{p}^{\prime}+1}(k)=g_{n_{p}^{\prime}+1}(0)+2 b k^{2}$ and determining the coefficient in the term with the second power of the wave vector magnitude $k$ is also given in [14].

It should be noted that the above-mentioned quantity $n_{0}$ for a threedimensional Ising-like system can be found by comparing the ratio of the critical amplitudes of the correlation length for $T>T_{c}$ and $T<T_{c}$ with 
data of numerical calculation from [19]. For $s=s^{*}$, we obtain $n_{0}=0.500$ (see [20]).

\section{The scheme of calculating the thermody- namic potential of a model in the tempera- ture region below $T_{c}$}

As in the case of $T>T_{c}$ [14, we shall calculate the thermodynamic potential $\Omega=-k T \ln \Xi$ by separating the contributions from short- and long-wave fluctuations of the order parameter $\left(\Omega_{C R}^{(-)}\right.$and $\Omega_{I G R}$, respectively). For $T<$ $T_{c}$, we have

$$
\Omega=\Omega_{\mu}+\Omega_{r}+\Omega_{C R}^{(-)}+\Omega_{I G R}
$$

Each of the terms is the contribution of a certain multiplier of the expression (11). Components $\Omega_{\mu}$ and $\Omega_{r}$ satisfy the same expressions as for $T>T_{c}$.

The term $\Omega_{C R}^{(-)}$in Eq. (17) corresponds to the contribution to the thermodynamic potential from the critical fluctuation regime. According to Eq. (11), we find

$$
\Omega_{C R}^{(-)}=-k T \sum_{n=1}^{n_{p}^{\prime}} N_{n} f_{n},
$$

where $N_{n}=N_{v} s^{-3 n}$. For the function $f_{n}\left(x_{n}, y_{n-1}\right)$, we obtain

$$
f_{n}=\frac{1}{2} \ln y_{n-1}+\frac{9}{4} y_{n-1}^{-2}+\frac{x_{n}^{2}}{4}+\ln U\left(0, x_{n}\right) .
$$

Here $U\left(0, x_{n}\right)$ is the parabolic cylinder function [21]. Expressions for arguments $x_{n}$ and $y_{n-1}$ are given in [22]. The argument $x_{n}$ is determined by the coefficients of the quartic fluctuation distribution in the $n$th layer of the phase space of the collective variables. The argument $y_{n-1}$ is the function of

$x_{n-1}$. It should be noted that taking into account Eq. (12), we arrive at the equalities

$$
\begin{aligned}
& s^{-\left(n_{p}^{\prime}+1\right)}=\left(\tilde{h}^{2}+h_{c m}^{2}\right)^{\frac{1}{d+2}}, \quad E_{1}^{n_{p}^{\prime}+1}=\left(\tilde{h}^{2}+h_{c m}^{2}\right)^{-\frac{1}{2}}, \\
& \tilde{\tau} E_{2}^{n_{p}^{\prime}+1}=-H_{c m}, \quad H_{c m}=-\tilde{\tau}\left(\tilde{h}^{2}+h_{c m}^{2}\right)^{-\frac{1}{2 p_{0}}}, \\
& E_{3}^{n_{p}^{\prime}+1}=H_{3 m}, \quad H_{3 m}=\left(\tilde{h}^{2}+h_{c m}^{2}\right)^{\frac{\Delta}{2 p_{0}}},
\end{aligned}
$$


where $p_{0}$ can be represented as $p_{0}=(d+2) \nu / 2(d=3$ is the space dimension, $\nu=\ln s^{*} / \ln E_{2}$ is the critical exponent of the correlation length), $\Delta=-\ln E_{3} / \ln E_{2}$ is the correction-to-scaling exponent. The renormalization group parameter $s^{*}=3.5977$ corresponds to the case when the value of $x_{n}$ at a fixed point vanishes $\left(x^{*}=0\right)$ [20]. For the $\rho^{4}$ model, we have $\nu=0.605, p_{0}=1.512, \Delta=0.465$. As a result of the calculation of $\Omega_{C R}^{(-)}$, we obtain

$$
\Omega_{C R}^{(-)}=-k T N_{v}\left(\gamma_{01}+\gamma_{02} \tau+\gamma_{03} \tau^{2}\right)+\Omega_{C R}^{(s)^{\prime}} .
$$

The formulas for the coefficients $\gamma_{0 l}$ appearing in the analytic part of this expression coincide with the corresponding quantities for $T>T_{c}$ (see [14]). Singular part has the form

$$
\Omega_{C R}^{(s)^{\prime}}=k T N_{v} \bar{\gamma}^{(-)} s^{-3\left(n_{p}^{\prime}+1\right)} .
$$

Here the coefficient

$$
\bar{\gamma}^{(-)}=\bar{\gamma}_{1}-\bar{\gamma}_{2} H_{c m}+\bar{\gamma}_{3} H_{c m}^{2}
$$

is the function of $H_{c m}$. The constant quantities $\bar{\gamma}_{l}$ are given in [14,20].

The term $\Omega_{I G R}$ in Eq. (17) is defined as

$$
\Omega_{I G R}=-k T \ln \Xi_{I G R}
$$

where the quantity $\Xi_{I G R}$ is presented in Eq. (15). The contribution to the thermodynamic potential from the inverse Gaussian regime (like the contribution to the thermodynamic potential from the limiting Gaussian regime for $T>T_{c}$ ) can be presented in the form

$$
\Omega_{I G R}=\Omega_{T R}^{(-)}+\Omega^{\prime \prime}
$$

Here term

$$
\Omega_{T R}^{(-)}=-k T N_{v} f_{n_{p}^{\prime}+1} s^{-3\left(n_{p}^{\prime}+1\right)}
$$

corresponds to the transition region from non-Gaussian to Gaussian fluctuations of the order parameter. The term $\Omega^{\prime \prime}$ will be calculated in Sec. IV by using the Gaussian distribution of fluctuations. The coefficient $f_{n_{p}^{\prime}+1}$ corresponds to $f_{n}$ from Eq. (19) for $n=n_{p}^{\prime}+1$. It is a function of $x_{n_{p}^{\prime}+1}$ and the quantity $y_{n_{p}^{\prime}}$, which in turn is expressed by $x_{n_{p}^{\prime}}$. The expression

$$
x_{n_{p}^{\prime}+m}=-\bar{x} E_{2}^{m-1} H_{c m}\left(1-\Phi_{q} E_{2}^{m-1} H_{c m}\right)^{-1 / 2}
$$


can be found on the basis of solutions of the recurrence relations, taking into account Eqs. (20). The quantities $\bar{x}$ and $\Phi_{q}$ are the same as in the case of $T>T_{c}$ (see [14]). It should be noted that the terms proportional to $H_{3 m} E_{3}^{m-1}$ are neglected when calculating $x_{n_{p}^{\prime}+m}$, Eq. (25), since the quantity $H_{3 m}$ from Eqs. (20) is small near the phase transition point, and $E_{3}<1$. Thus, we disregard the corrections to scaling in the present calculations.

Calculating $\Omega_{T R}^{(-)}$, Eq. (24), we single out the transition region from nonGaussian to Gaussian fluctuations of the order parameter, which corresponds to one layer of the phase space of the collective variables. As follows from the results of [7], singling out the transition region at $T<T_{c}$ is optional. Introducing this region for temperatures $T<T_{c}$, we followed the thermodynamic potential calculation scheme given in [14 for $T>T_{c}$. It does not diminish the generalization of our study in any way but it does allow us to unify the calculation scheme.

\section{Forming inverse Gaussian distribution of fluctuations}

Let us calculate the contribution to the thermodynamic potential $\Omega^{\prime \prime}=$ $-k T \ln \Xi^{\prime \prime}$ appearing in Eq. (23). For that we need to calculate the corresponding component of the grand partition function

$$
\Xi^{\prime \prime}=2^{\left(N_{n_{p}^{\prime}+2}-1\right) / 2}\left[Q\left(P_{n_{p}^{\prime}+1}\right)\right]^{N_{n_{p}^{\prime}+2}} \Xi_{n_{p}^{\prime}+2},
$$

where

$$
\begin{aligned}
& \Xi_{n_{p}^{\prime}+2}=\int(d \rho)^{N_{n_{p}^{\prime}+2}} \exp \left[\tilde{h} \sqrt{N_{v}} \rho_{0}\right. \\
& -\frac{1}{2} \sum_{\mathbf{k} \in \mathcal{B}_{n_{p}^{\prime}+2}} g_{n_{p}+2}(k) \rho_{\mathbf{k}} \rho_{-\mathbf{k}} \\
& \left.-\frac{a_{4}^{\left(n_{p}^{\prime}+2\right)}}{4 !} N_{n_{p}^{\prime}+2}^{-1} \sum_{\substack{\mathbf{k}_{1}, \ldots, \mathbf{k}_{4} \\
\mathbf{k}_{i} \in \mathcal{B}_{n_{p}^{\prime}+2}}} \rho_{\mathbf{k}_{1}} \cdots \rho_{\mathbf{k}_{4}} \delta_{\mathbf{k}_{1}+\cdots+\mathbf{k}_{4}}\right] .
\end{aligned}
$$

For the coefficients $g_{n_{p}^{\prime}+2}(k)$ and $a_{4}^{\left(n_{p}^{\prime}+2\right)}$, we obtain

$$
g_{n_{p}^{\prime}+2}(k)=g_{n_{p}^{\prime}+2}(0)+2 b k^{2}
$$




$$
\begin{aligned}
& g_{n_{p}^{\prime}+2}(0)=s^{-2\left(n_{p}^{\prime}+2\right)} r_{n_{p}^{\prime}+2}, \\
& a_{4}^{\left(n_{p}^{\prime}+2\right)}=s^{-4\left(n_{p}^{\prime}+2\right)} u_{n_{p}^{\prime}+2} .
\end{aligned}
$$

The quantities $r_{n_{p}^{\prime}+2}$ and $u_{n_{p}^{\prime}+2}$ are determined by the relations

$$
\begin{aligned}
& r_{n_{p}^{\prime}+2}=q\left(-1-E_{2} H_{c m}\right), \\
& u_{n_{p}^{\prime}+2}=u^{*}\left(1-\Phi_{q} E_{2} H_{c m}\right) .
\end{aligned}
$$

Here $u^{*}=\left[q\left(1-s^{-2}\right) \sqrt{3} U\left(x^{*}\right)\left[1+3 /\left(2 y^{*}\right)^{2}\right]\right]^{2}$ is the quantity $u_{n}=s^{4 n} a_{4}^{(n)}$ at a fixed point (see [14]). The quantity $\tilde{h}$ appearing in Eq. (27) is characterized by the chemical potential. It corresponds to an introduction of an external magnetic field in a three-dimensional Ising-like system [23 25].

The coefficient $u_{n_{p}^{\prime}+2}$ remains positive for arbitrary values of temperature and chemical potential, so that the integral in Eq. (27) converges. The behavior of the coefficient $r_{n_{p}^{\prime}+2}$, as well as of the analogous quantity $r_{n_{p}+2}$ for $T>T_{c}$, depending on the variation of $\tau$ and $M$ is shown in Fig. 1. As is seen from Fig. 1 $\mathrm{b}$, the coefficient $r_{n_{p}+2}$ decreases with increasing magnitude of the chemical potential $|M|$ and becomes negative for small values of the relative temperature $\tau$. The coefficient $r_{n_{p}^{\prime}+2}$ takes on negative values for all temperatures $T<T_{c}$ (see Fig. 1 1a). As a result, using the Gaussian approximation does not make sense when calculating $\Xi_{n_{p}^{\prime}+2}$, Eq. (27). The situation can be improved by introducing in the expression (27) the substitution of variables

$$
\rho_{\mathbf{k}}=\eta_{\mathbf{k}}+\sqrt{N_{v}} \sigma_{-} \delta_{\mathbf{k}}
$$

where $\sigma_{-}$is a constant quantity. As a result of the substitution (28), the expression (27) would take the form

$$
\begin{aligned}
& \Xi_{n_{p}^{\prime}+2}=e^{N_{v} E_{0}\left(\sigma_{-}\right)} \int(d \eta)^{N_{n_{p}^{\prime}+2} \exp }\left[A_{0}^{\prime} \sqrt{N_{v}} \eta_{0}\right. \\
& -\frac{1}{2} \sum_{\mathbf{k} \in \mathcal{B}_{n_{p}^{\prime}+2}} \overline{g^{\prime}}(k) \eta_{\mathbf{k}} \eta_{-\mathbf{k}}-\frac{\overline{b^{\prime}}}{6} N_{n_{p}^{\prime}+2}^{-1 / 2} \sum_{\substack{\mathbf{k}_{1}, \ldots, \mathbf{k}_{3} \\
\mathbf{k}_{i} \in \mathcal{B}_{n_{p}^{\prime}+2}}} \eta_{\mathbf{k}_{1}} \cdots \eta_{\mathbf{k}_{3}} \delta_{\mathbf{k}_{1}+\cdots+\mathbf{k}_{3}} \\
& \left.-\frac{\overline{a^{\prime}}}{24} N_{n_{p}^{\prime}+2}^{-1} \sum_{\substack{\mathbf{k}_{1}, \ldots, \mathbf{k}_{4} \\
\mathbf{k}_{i} \in \mathbf{B}_{n_{p}+2}}} \eta_{\mathbf{k}_{1}} \cdots \eta_{\mathbf{k}_{4}} \delta_{\mathbf{k}_{1}+\cdots+\mathbf{k}_{4}}\right] \text {. }
\end{aligned}
$$

Here

$$
E_{0}\left(\sigma_{-}\right)=\tilde{h} \sigma_{-}-\frac{r_{n_{p}^{\prime}+2}}{2} s^{-2\left(n_{p}^{\prime}+2\right)} \sigma_{-}^{2}-\frac{u_{n_{p}^{\prime}+2}}{24} s^{-\left(n_{p}^{\prime}+2\right)} \sigma_{-}^{4}
$$




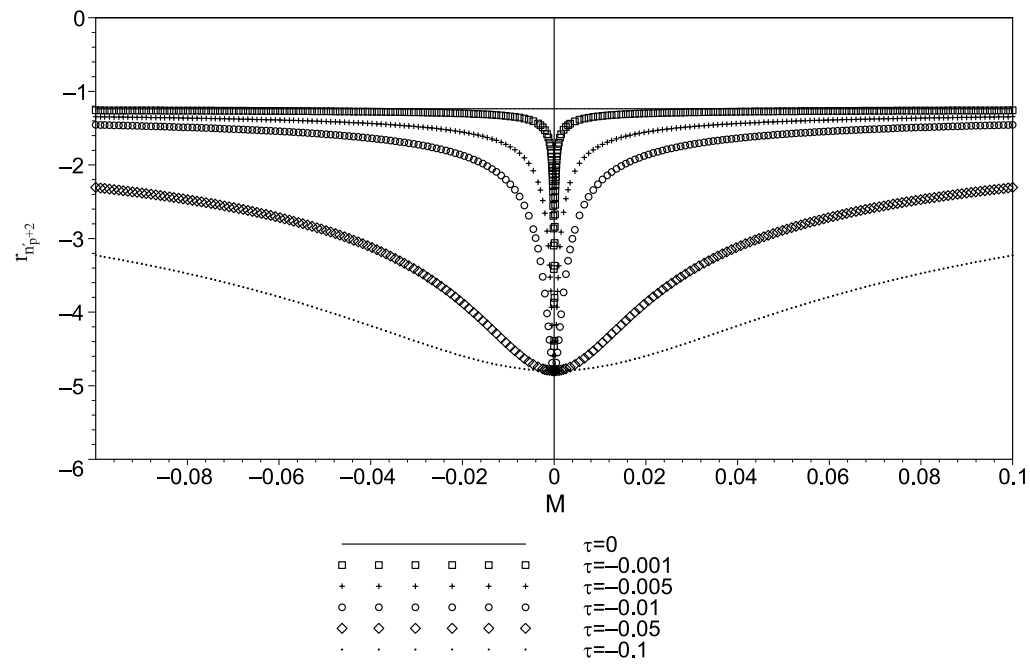

a

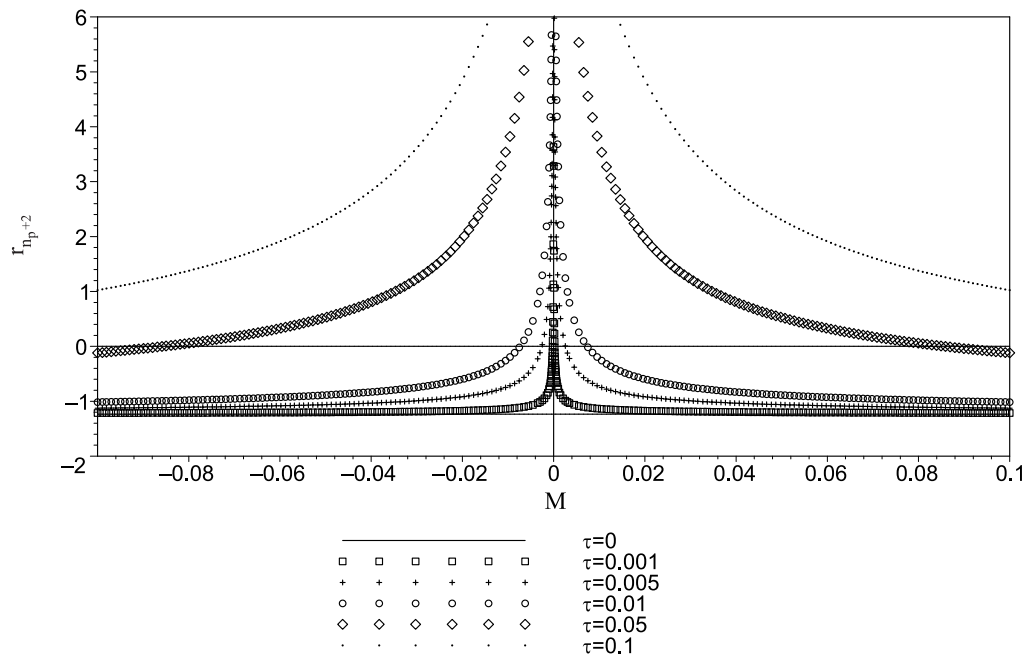

$\mathrm{b}$

Figure 1: Dependence of the quantity $r_{n_{p}^{\prime}+2}$ in the case of $T<T_{c}$ (figure a) and the analogous quantity $r_{n_{p}+2}$ for $T>T_{c}$ (figure b) on the chemical potential $M$ for various values of the relative temperature $\tau$. 
For the coefficients $A_{0}^{\prime}, \bar{g}^{\prime}(k), \overline{b^{\prime}}$, and $\bar{a}_{4}^{\prime}$, we obtain the following expressions:

$$
\begin{aligned}
& A_{0}^{\prime}=\tilde{h}-r_{n_{p}^{\prime}+2} s^{-2\left(n_{p}^{\prime}+2\right)} \sigma_{-}-\frac{u_{n_{p}^{\prime}+2}}{6} s^{-\left(n_{p}^{\prime}+2\right)} \sigma_{-}^{3}, \\
& \bar{g}^{\prime}(k)=\bar{g}^{\prime}(0)+2 b k^{2}, \\
& \overline{g^{\prime}}(0)=r_{n_{p}^{\prime}+2} s^{-2\left(n_{p}^{\prime}+2\right)}+\frac{u_{n_{p}^{\prime}+2}}{2} s^{-\left(n_{p}^{\prime}+2\right)} \sigma_{-}^{2}, \\
& \bar{b}^{\prime}=u_{n_{p}^{\prime}+2} s^{-5\left(n_{p}^{\prime}+2\right) / 2} \sigma_{-}, \quad{\overline{a^{\prime}}}_{4}=u_{n_{p}^{\prime}+2} s^{-4\left(n_{p}^{\prime}+2\right)} .
\end{aligned}
$$

Similarly to the case of $T>T_{c}$, we find the shift quantity $\sigma_{-}$from the condition

$$
\frac{\partial E_{0}\left(\sigma_{-}\right)}{\partial \sigma_{-}}=0
$$

Taking into account Eq. (30) and the expression for $A_{0}^{\prime}$ [see Eqs. (31)], we obtain the equation

$$
A_{0}^{\prime}=0 \text {. }
$$

The solution of this equation can be presented in the form

$$
\sigma_{-}=\sigma_{0}^{\prime} s^{-\left(n_{p}^{\prime}+2\right) / 2}
$$

For the quantity $\sigma_{0}^{\prime}$, we have a cubic equation

$$
\left(\sigma_{0}^{\prime}\right)^{3}+p^{\prime} \sigma_{0}^{\prime}+q^{\prime}=0
$$

with coefficients

$$
p^{\prime}=6 \frac{r_{n_{p}^{\prime}+2}}{u_{n_{p}^{\prime}+2}}, \quad q^{\prime}=-6 \frac{s^{5 / 2}}{u_{n_{p}^{\prime}+2}} \frac{\tilde{h}}{\left(\tilde{h}^{2}+h_{c m}^{2}\right)^{1 / 2}} .
$$

In general case, the quantities $p^{\prime}$ and $q^{\prime}$ are functions of temperature and chemical potential. The form of solutions of the equation (34) depends on the sign of the discriminant

$$
Q=\left(p^{\prime} / 3\right)^{3}+\left(q^{\prime} / 2\right)^{2} .
$$

Let us write the expression determining the magnitude of the chemical potential $M_{q}$ at $Q=0$. It is defined as

$$
M_{q}=\left[-\frac{8 r_{n_{p}^{\prime}+2}^{3}\left(1+\alpha_{m q}^{2}\right)}{9 u_{n_{p}^{\prime}+2} s^{5} \beta W(0)}\right]^{1 / 2} h_{c m} .
$$


Then, by substituting the quantity $\alpha_{m q}=M_{q}(\beta W(0))^{1 / 2} / h_{c m}$ in the formula (36), we find

$$
M_{q}=\left[-\frac{8 r_{n_{p}^{\prime}+2}^{3}}{9 u_{n_{p}^{\prime}+2} s^{5} \beta W(0)\left(1+\frac{8 r_{n_{p}^{\prime}+2}^{3}}{9 u_{n_{p}^{\prime}+s^{5}}}\right)}\right]^{1 / 2} h_{c m} .
$$

For $Q>0$, in accordance with Cardano's formula, the real solution $\sigma_{0}^{\prime}$ of the equation (34) assumes the following form:

$$
\sigma_{0 b}^{\prime}=A+B, \quad A=\left(-q^{\prime} / 2+Q^{1 / 2}\right)^{1 / 3}, \quad B=\left(-q^{\prime} / 2-Q^{1 / 2}\right)^{1 / 3} .
$$

For $Q<0$, we have three real solutions (the quantity $\sigma_{0}^{\prime}$ takes on three possible real values)

$$
\begin{aligned}
& \sigma_{01}^{\prime}=2\left(-p^{\prime} / 3\right)^{1 / 2} \cos \left(\alpha_{r} / 3\right), \\
& \sigma_{02,03}^{\prime}=-2\left(-p^{\prime} / 3\right)^{1 / 2} \cos \left(\alpha_{r} / 3 \pm \pi / 3\right),
\end{aligned}
$$

where $\alpha_{r}$ is determined from the relation

$$
\cos \alpha_{r}=-\frac{q^{\prime}}{2\left(-p^{\prime} / 3\right)^{3 / 2}} .
$$

The dependence of the roots of the cubic equation (34) on the chemical potential $M$ for $T<T_{c}$ is demonstrated by the curves in Fig. 2,

It follows from the results of calculations that the coefficient of the quadratic term in the exponent of the expression (29) takes on a large value in comparison with the coefficients of other terms. We single out in Eq. (29) the terms with $k=0$ and perform integration with respect to variables $\eta_{\mathbf{k}}$ with $k \neq 0$ using the Gaussian distribution of fluctuations as the basis one. In a zero-order approximation at $k \neq 0$, we arrive at the expression

$$
\Xi_{n_{p}^{\prime}+2}=e^{N_{v} E_{0}\left(\sigma_{-}\right)} \prod_{\substack{k \neq 0 \\ \mathbf{k} \in \mathcal{B}_{n_{p}^{\prime}+2}}}\left(\pi / \bar{g}^{\prime}(k)\right)^{1 / 2} \Xi_{n_{p}^{\prime}+2}^{(0)} .
$$

Here

$$
\begin{aligned}
& \Xi_{n_{p}^{\prime}+2}^{(0)}=\int_{-\infty}^{+\infty} d \eta_{0} \exp \left[A_{0}^{\prime} \sqrt{N_{v}} \eta_{0}-\frac{1}{2} \overline{g^{\prime}}(0) \eta_{0}^{2}-\frac{\overline{b^{\prime}}}{6} N_{n_{p}^{\prime}+2}^{-1 / 2} \eta_{0}^{3}\right. \\
& \left.-\frac{\bar{a}_{4}^{\prime}}{24} N_{n_{p}^{\prime}+2}^{-1} \eta_{0}^{4}\right] .
\end{aligned}
$$




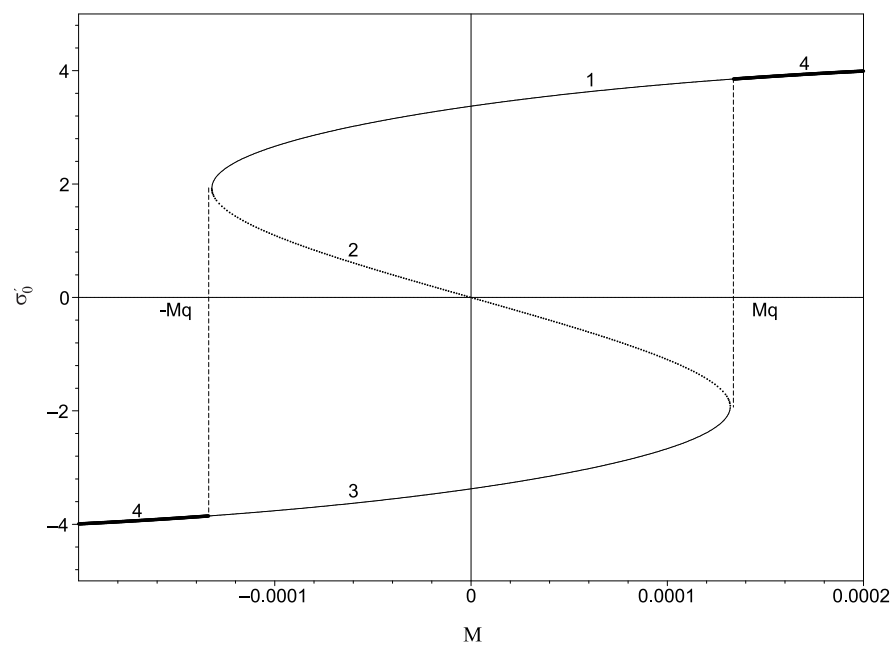

Figure 2: Solutions of the cubic equation (34) as functions of the chemical the potential $M$ for $\tau=-0.005$. Curves $1,2,3$, and 4 correspond to solutions $\sigma_{0}^{\prime}=\sigma_{01}^{\prime}, \sigma_{0}^{\prime}=\sigma_{02}^{\prime}, \sigma_{0}^{\prime}=\sigma_{03}^{\prime}$, and $\sigma_{0}^{\prime}=\sigma_{0 b}^{\prime}$, respectively.

Eliminating the cubic term in the exponent of the expression for $\Xi_{n_{p}^{\prime}+2}^{(0)}$ by the substitution of the variable

$$
\eta_{0}=\rho_{0}-\sqrt{N_{v}} \sigma_{-}
$$

and using the steepest-descent method in integrating with respect to the variable $\rho_{0}$, we obtain the following formula for the thermodynamic potential contribution corresponding to $\Xi_{n_{p}^{\prime}+2}$ from Eq. (39):

$$
\Omega_{n_{p}^{\prime}+2}=-k T N_{v} E_{0}\left(\sigma_{-}\right)-\frac{1}{2} k T N_{n_{p}^{\prime}+2} \ln \pi+\frac{1}{2} k T \sum_{\substack{k \neq 0 \\ \mathbf{k} \in \mathcal{B}_{n_{p}^{\prime}+2}}} \ln \overline{g^{\prime}}(k) .
$$

Here

$$
E_{0}\left(\sigma_{-}\right)=e_{0}^{(-)} \tilde{h} s^{-\left(n_{p}^{\prime}+1\right) / 2}-e_{2}^{(-)} s^{-3\left(n_{p}^{\prime}+1\right)},
$$

and the coefficients $e_{0}^{(-)}$and $e_{2}^{(-)}$satisfy the relations

$$
e_{0}^{(-)}=\sigma_{0}^{\prime} s^{-1 / 2}, \quad e_{2}^{(-)}=\frac{\left(\sigma_{0}^{\prime}\right)^{2}}{2} s^{-3}\left[r_{n_{p}^{\prime}+2}+\frac{u_{n_{p}^{\prime}+2}}{12}\left(\sigma_{0}^{\prime}\right)^{2}\right] .
$$

It should be noted that the change of the variable $\eta_{0}$ [see Eq. (41)] in the expression (40) and the substitution $\rho_{0}=\sqrt{N_{v}} \rho$ lead to an appearance of a 
sharp maximum of the integrand at the point $\bar{\rho}$. The extremum condition of the integrand, from which $\bar{\rho}$ is determined, and the representation $\bar{\rho}=$ $\overline{\rho^{\prime \prime}} s^{-\left(n_{p}^{\prime}+2\right) / 2}$ lead to the same cubic equation for $\overline{\rho^{\prime \prime}}$ (with the same coefficients) as for $\sigma_{0}^{\prime}$ [see Eq. (34) ]. The quantities $\bar{\rho}^{\prime \prime}$ and $\sigma_{0}^{\prime}$ as well as $\bar{\rho}$ and $\sigma_{-}$take on the same values. This allows us to represent the integrand $E_{0}(\rho)$ at the point $\bar{\rho}$ as $E_{0}\left(\sigma_{-}\right)$[see Eq. (43)] with coefficients from Eqs. (44), where the quantity $\bar{\rho}^{\prime \prime}$ is replaced by $\sigma_{0}^{\prime}$. Replacing summation over $\mathbf{k} \in \mathcal{B}_{n_{p}^{\prime}+2}$ by integration, we arrive at the following formula for the last term in Eq. (42):

$$
\begin{aligned}
& \frac{1}{2} \sum_{\mathbf{k} \in \mathcal{B}_{n_{p}^{\prime}+2}} \ln \bar{g}^{\prime}(k)=k T N_{n_{p}^{\prime}+2}\left\{\frac{1}{2} \ln \left(1+a_{I}^{2}\right)-\left(n_{p}^{\prime}+2\right) \ln s\right. \\
& \left.+\frac{1}{2} \ln r_{R}^{\prime}-\frac{1}{3}+\frac{1}{a_{I}^{2}}-\frac{1}{a_{I}^{3}} \arctan a_{I}\right\} \\
& a_{I}=\frac{\pi}{c}\left(\frac{2 b}{r_{R}^{\prime}}\right)^{1 / 2}, \quad r_{R}^{\prime}=r_{n_{p}^{\prime}+2}+\frac{u_{n_{p}^{\prime}+2}}{2}\left(\sigma_{0}^{\prime}\right)^{2} .
\end{aligned}
$$

Now, using Eq. (42), we can write the thermodynamic potential contribution corresponding to $\Xi^{\prime \prime}$ from Eq. (26) as the sum of two terms

$$
\Omega^{\prime \prime}=\Omega_{0}^{(-)}+\Omega_{I}^{\prime}
$$

The component

$$
\Omega_{0}^{(-)}=-k T N_{v} E_{0}\left(\sigma_{-}\right)
$$

is related to the shift of the variable $\rho_{0}$, which is determined by the quantity that is the solution of the cubic equation. For another component $\Omega_{I}^{\prime}$, we obtain

$$
\Omega_{I}^{\prime}=-k T N_{n_{p}^{\prime}+2} f_{I},
$$

where

$$
\begin{aligned}
& f_{I}=\left(-\frac{1}{2} \ln 3+2 \ln s+\frac{1}{2} \ln u_{n_{p}^{\prime}+1}-\ln r_{R}^{\prime}-\ln U\left(x_{n_{p}^{\prime}+1}\right)\right. \\
& \left.-\frac{3}{4} y_{n_{p}^{\prime}+1}^{-2}-f_{I}^{\prime \prime}\right) / 2 .
\end{aligned}
$$

The quantity $r_{R}^{\prime}$ is given in Eqs. (45). The remaining quantities in Eq. (47) are defined as

$$
u_{n_{p}^{\prime}+1}=u^{*}\left(1-\Phi_{q} H_{c m}\right)
$$




$$
\begin{aligned}
& x_{n_{p}^{\prime}+1}=-\bar{x} H_{c m}\left(1-\Phi_{q} H_{c m}\right)^{-1 / 2}, \\
& y_{n_{p}^{\prime}+1}=s^{3 / 2} U\left(x_{n_{p}^{\prime}+1}\right)\left(3 / \varphi\left(x_{n_{p}^{\prime}+1}\right)\right)^{1 / 2}, \\
& f_{I}^{\prime \prime}=\ln \left(1+a_{I}^{2}\right)-\frac{2}{3}+\frac{2}{a_{I}^{2}}-\frac{2}{a_{I}^{3}} \arctan a_{I} .
\end{aligned}
$$

Taking into account $\Omega_{T R}^{(-)}$, Eq. (24), and $\Omega^{\prime \prime}$, Eq. (46), we can find the contribution to the thermodynamic potential $\Omega_{I G R}$, Eq. (23).

Let us add up the above-obtained contributions to the thermodynamic potential near the critical point at $T<T_{c}$. According to Eq. (17), the thermodynamic potential of a cell fluid model is represented as the sum of several terms. Singling out the analytic and nonanalytic parts of the thermodynamic potential, we obtain the following complete expression equivalent to Eq. (17):

$$
\Omega=\Omega_{a}+\Omega_{s}^{(-)}+\Omega_{0}^{(-)} .
$$

The analytic contribution has the form

$$
\Omega_{a}=-k T N_{v}\left(\gamma_{01}-\gamma_{02}|\tau|+\gamma_{03}|\tau|^{2}\right)+\Omega_{01}
$$

where the formulas for $\gamma_{01}, \gamma_{02}, \gamma_{03}$, and $\Omega_{01}=-k T N_{v}\left[E_{\mu}+\gamma_{a}\right]$ are the same as in the case of $T>T_{c}$. The nonanalytic contribution satisfies the relation

$$
\Omega_{s}^{(-)}=-k T N_{v} \gamma_{s}^{(-)}\left(\tilde{h}^{2}+h_{c m}^{2}\right)^{\frac{d}{d+2}}
$$

Here

$$
\gamma_{s}^{(-)}=f_{n_{p}^{\prime}+1}-\bar{\gamma}^{(-)}+f_{I} / s^{3}
$$

The term

$$
\begin{aligned}
& \Omega_{0}^{(-)}=-k T N_{v}\left[e_{0}^{(-)} \tilde{h}\left(\tilde{h}^{2}+h_{c m}^{2}\right)^{\frac{d-2}{2(d+2)}}\right. \\
& \left.-e_{2}^{(-)}\left(\tilde{h}^{2}+h_{c m}^{2}\right)^{\frac{d}{d+2}}\right]
\end{aligned}
$$

is characterized by the coefficients $e_{0}^{(-)}$and $e_{2}^{(-)}$given in Eqs. (44).

Comparing the temperature regions below $T_{c}$ (the present calculations) and above $T_{c}$ (see [14]), it should be noted that the value of the system's point of exit from the critical fluctuation regime for $T<T_{c}$ is somewhat smaller than the analogous value for $T>T_{c}$. For this reason, we have different temperature measurement scales for each temperature interval. Using the 
obtained results, we can write the general formula for the thermodynamic potential

$$
\Omega=\Omega_{a}+\Omega_{s}+\Omega_{0},
$$

where the analytic part $\Omega_{a}$ is common to both temperature intervals, and the contributions $\Omega_{s}$ and $\Omega_{0}$ are given by the expressions

$$
\Omega_{s}=\left\{\begin{array}{lll}
\Omega_{s}^{(-)}, & \text {at } & T<T_{c} \\
\Omega_{s}^{(+)}, & \text {at } T \geq T_{c}
\end{array}\right.
$$

and

$$
\Omega_{0}= \begin{cases}\Omega_{0}^{(-)}, & \text {at } T<T_{c} \\ \Omega_{0}^{(+)}, & \text {at } T \geq T_{c},\end{cases}
$$

respectively. Thus, we have the general functional representation of the thermodynamic potential, which depends on both the temperature and the chemical potential and is valid for both sides of the critical temperature.

\section{Equation of state of the model at $T<T_{c}$ with allowance for the order parameter fluctuations}

We shall use the relation

$$
\bar{N}=\frac{\partial \ln \Xi}{\partial \beta \mu}
$$

which makes it possible to express the chemical potential in terms of the average number of particles $\bar{N}$ or in terms of the average density

$$
\bar{n}=\frac{\bar{N}}{N_{v}}=\left(\frac{\bar{N}}{V}\right) v
$$

Here $v$ is the volume of the elementary cell.

On the basis of Eqs. (55) and (56), taking into account Eq. (49), we obtain

$$
\bar{n}=\bar{n}_{a}+n_{s}^{(-)}+n_{0}^{(-)}
$$


where

$$
\begin{aligned}
& \bar{n}_{a}=\frac{\partial E_{\mu}}{\partial \beta \mu}, \\
& n_{s}^{(-)}=\frac{\partial}{\partial \beta \mu}\left[\gamma_{s}^{(-)}\left(\tilde{h}^{2}+h_{c m}^{2}\right)^{\frac{d}{d+2}}\right], \\
& n_{0}^{(-)}=\frac{\partial}{\partial \beta \mu}\left[e_{0}^{(-)} \tilde{h}\left(\tilde{h}^{2}+h_{c m}^{2}\right)^{\frac{d-2}{2(d+2)}}-e_{2}^{(-)}\left(\tilde{h}^{2}+h_{c m}^{2}\right)^{\frac{d}{d+2}}\right] .
\end{aligned}
$$

In accordance with Eq. (157) and expressions in Appendix A, the total contribution to the average density assumes the following form:

$$
\bar{n}=-M-\tilde{a}_{1}+\frac{1}{\beta W(0)} a_{34}+\sigma_{00}^{(-)}\left(\tilde{h}^{2}+h_{c m}^{2}\right)^{\frac{d-2}{2(d+2)}} .
$$

For the coefficient $\sigma_{00}^{(-)}$, we have

$$
\begin{aligned}
& \sigma_{00}^{(-)}=e_{0}^{(-)} \frac{1}{(\beta W(0))^{1 / 2}}\left(1+\frac{d-2}{d+2} \frac{\tilde{h}^{2}}{\tilde{h}^{2}+h_{c m}^{2}}\right) \\
& +e_{00}^{(-)} \frac{\tilde{h}}{\left(\tilde{h}^{2}+h_{c m}^{2}\right)^{1 / 2}}+e_{02}^{(-)} .
\end{aligned}
$$

Here the quantity $e_{0}^{(-)}$is defined in Eqs. (44), and for $e_{00}^{(-)}$and $e_{02}^{(-)}$, we find expressions

$$
\begin{aligned}
& e_{00}^{(-)}=\frac{2 d}{d+2} \frac{1}{(\beta W(0))^{1 / 2}}\left(\gamma_{s}^{(-)}-e_{2}^{(-)}\right), \\
& e_{02}^{(-)}=\left(\frac{\partial \gamma_{s}^{(-)}}{\partial \beta \mu}-\frac{\partial e_{2}^{(-)}}{\partial \beta \mu}\right)\left(\tilde{h}^{2}+h_{c m}^{2}\right)^{1 / 2} .
\end{aligned}
$$

The first two terms on the right-hand side of the equality (60) depend only on the variable $\alpha_{m}=\tilde{h} / h_{c m}$. For quantity $\sigma_{00}^{(-)}$to be a function of only $\alpha_{m}$, it is necessary for term $e_{02}^{(-)}$to be such a function. This can be verified by direct differentiation of the quantities $\gamma_{s}^{(-)}$and $e_{2}^{(-)}$with respect to $\beta \mu$ (see Appendix B).

Taking into account the relations (10) for $\tilde{a}_{1}, d(0)$, and $\tilde{a}_{2}$, we rewrite the equation (59) in the following form:

$$
\bar{n}=n_{g}-M+\sigma_{00}^{(-)}\left(\tilde{h}^{2}+h_{c m}^{2}\right)^{\frac{d-2}{2(d+2)}} .
$$


Here

$$
n_{g}=-a_{1}-a_{2} a_{34}+\frac{a_{4}}{3} a_{34}^{3}
$$

The coefficient $\sigma_{00}^{(-)}$is given in Appendix B by Eq. (B.10). The quantity $\tilde{h}$ as a function of $M$ is defined in Eq. (13).

The nonlinear equation (62) describes the relationship between the density $\bar{n}$ and the chemical potential $M$ given in Eqs. (10). It can be presented in the form

$$
\bar{n}-n_{g}+M=\left(\frac{M b_{1}^{(-)}}{b_{2}^{(-)}}\right)^{1 / 5} \sigma_{00}^{(-)}
$$

or

$$
b_{3}^{(-)} M^{1 / 5}=\bar{n}-n_{g}+M
$$

where

$$
\begin{aligned}
& b_{1}^{(-)}=(\beta W(0))^{1 / 2}, \quad b_{2}^{(-)}=\frac{\alpha_{m}}{\left(1+\alpha_{m}^{2}\right)^{1 / 2}}, \\
& b_{3}^{(-)}=\left(\frac{b_{1}^{(-)}}{b_{2}^{(-)}}\right)^{1 / 5} \sigma_{00}^{(-)} .
\end{aligned}
$$

Using Eq. (65), we can express the chemical potential $M$ in terms of the average density $\bar{n}$. Considering that $M \ll 1$ and neglecting the last term on the right-hand side of the equation (65), we obtain an approximate formula

$$
M=\left(\frac{\bar{n}-n_{g}}{\sigma_{00}^{(-)}}\right)^{5} \frac{b_{2}^{(-)}}{(\beta W(0))^{1 / 2}}
$$

or (taking into account the expressions for $b_{2}^{(-)}$and $\alpha_{m}$ )

$$
M=\frac{h_{c m}}{(\beta W(0))^{1 / 2}}\left[\left(\frac{\bar{n}-n_{g}}{\sigma_{00}^{(-)}}\right)^{10} \frac{1}{h_{c m}^{2}}-1\right]^{1 / 2} .
$$

The equation (65) allows us to trace the evolution of the average density $\bar{n} \approx n_{g}+b_{3}^{(-)} M^{1 / 5}$ with the variation of $M$ for various negative values of the relative temperature $\tau$ (see Figs. 3 and 4 ).

Let us evaluate the values of critical exponents characterizing the behavior of the density $\bar{n}$ as a function of temperature and chemical potential $M$ [see Eq. (62), where the external field $\tilde{h}$ (which is proportional to 


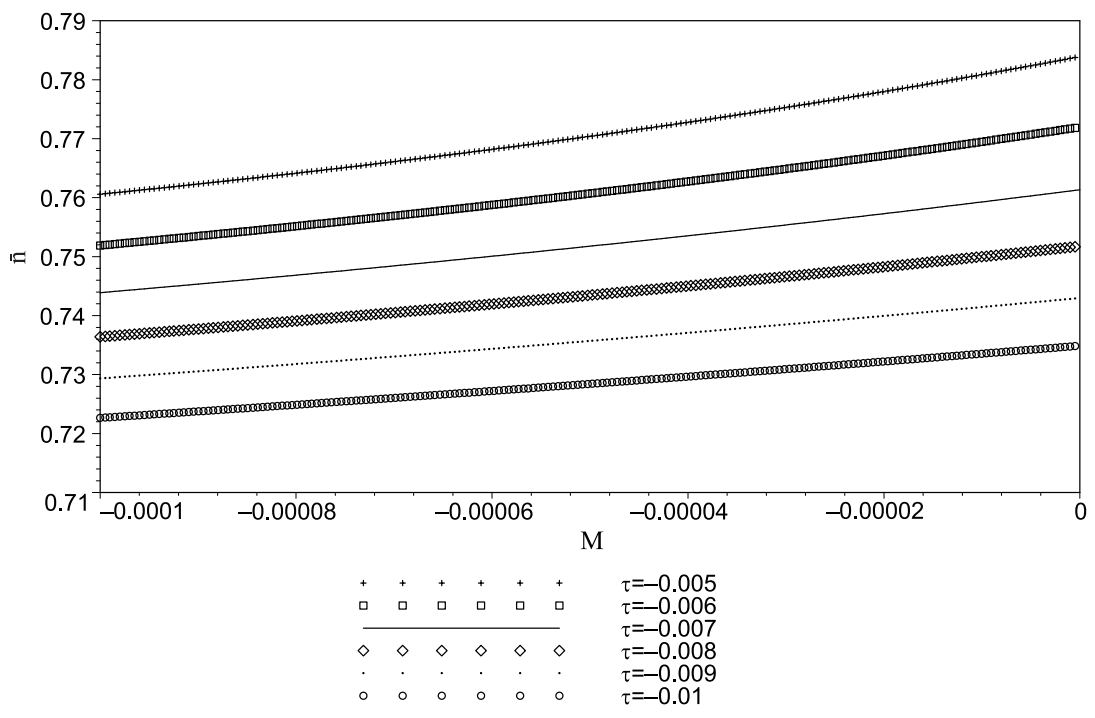

Figure 3: Density $\bar{n}$ at negative values of the chemical potential $M$ for some values of $\tau$.

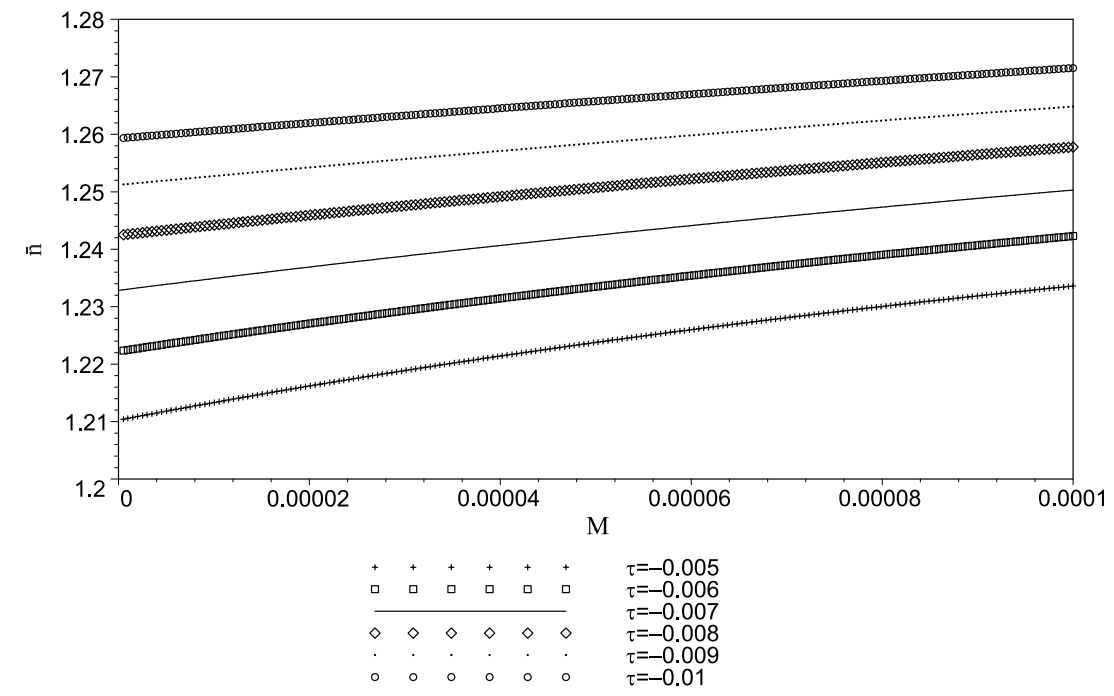

Figure 4: Density $\bar{n}$ at positive values of the chemical potential of $M$ for various $\tau$. 
$M)$ and the temperature field $h_{c m}$ are given by the expressions (13) and (14), respectively]. In the case of $M=0$ and $T \neq T_{c}$, the equation (62) can be rewritten as $\bar{n}=\bar{n}_{c}+\sigma_{00}^{(-)}(M=0) \tilde{\tau}_{1}^{\beta}$. Here the critical exponent $\beta=\nu / 2=0.302$ is determined by the critical exponent of the correlation length $\nu=\ln s^{*} / \ln E_{2}=0.605$, and $\bar{n}_{c}=n_{g}$ is the critical density. In the case of $M \neq 0$ and $T=T_{c}$, the equation (62) can be represented as $\bar{n} \approx \bar{n}_{c}+\sigma_{00}^{(-)}\left(T_{c}\right) \tilde{h}^{1 / \delta}$, where the critical exponent $\delta$ is equal to 5 . Thus, the values of the critical exponents $\nu, \beta$, and $\delta$ are nonclassical.

Taking into account the relations $P V=k T \ln \Xi$ and $\Omega=-k T \ln \Xi$ as well as Eq. (49) and $V=N_{v} v$, we obtain the following the equation of state at $T<T_{c}$ :

$$
\begin{aligned}
& \frac{P v}{k T}=P_{a}^{(-)}(T)+E_{\mu}+\left(\gamma_{s}^{(-)}-e_{2}^{(-)}\right)\left(\tilde{h}^{2}+h_{c m}^{2}\right)^{\frac{d}{d+2}} \\
& +e_{0}^{(-)} \tilde{h}\left(\tilde{h}^{2}+h_{c m}^{2}\right)^{\frac{d-2}{2(d+2)}}, \\
& P_{a}^{(-)}(T)=\gamma_{a}+\gamma_{01}-\gamma_{02}|\tau|+\gamma_{03}|\tau|^{2}, \\
& \gamma_{a}=f_{W}^{\prime}-a_{0}+\frac{1}{4} \ln \left(\frac{3}{u_{0}}\right)+\frac{x^{2}}{4}+\ln U(0, x), \\
& f_{W}^{\prime}=-\frac{1}{2} \ln \left[1-\left(a^{\prime}\right)^{2}\right]+\frac{1}{3}+\frac{1}{\left(a^{\prime}\right)^{2}}-\frac{1}{2\left(a^{\prime}\right)^{3}} \ln \left|\frac{1+a^{\prime}}{1-a^{\prime}}\right| .
\end{aligned}
$$

Here $a^{\prime}=\pi(2 b)^{1 / 2} / c$. The quantity $M$ appearing in the expression for $E_{\mu}$ [see Eqs. (10)] is given by Eq. (67). For $\tilde{h}$, taking into account Eq. (67), we find

$$
\tilde{h}=\left(\frac{\bar{n}-n_{g}}{\sigma_{00}^{(-)}}\right)^{5} b_{2}^{(-)},
$$

and the quantity $h_{c m}$ is defined in Eq. (14). Determining the sum $\tilde{h}^{2}+h_{c m}^{2}$ from Eq. (62) and substituting it in the expression for $P v /(k T)$ [see Eqs. (68)], we can rewrite the equation of state in the following form:

$$
\begin{aligned}
& \frac{P v}{k T}=P_{a}^{(-)}(T)+E_{\mu}+\left(\frac{\bar{n}-n_{g}}{\sigma_{00}^{(-)}}\right)^{6}\left[e_{0}^{(-)} \frac{\alpha_{m}}{\left(1+\alpha_{m}^{2}\right)^{1 / 2}}\right. \\
& \left.+\gamma_{s}^{(-)}-e_{2}^{(-)}\right] .
\end{aligned}
$$


The quantities $P_{a}^{(-)}(T), E_{\mu}$, and $\sigma_{00}^{(-)}$are given in Eqs. (68), (10), and (B.10), respectively. The coefficients $e_{0}^{(-)}, e_{2}^{(-)}$, and $\gamma_{s}^{(-)}$are defined by the relations (44) and (52). The quantities

$$
\begin{aligned}
& a_{1}=-\frac{T_{1}(v, p)}{T_{0}(v, p)}, \quad a_{2}=-\frac{T_{2}(v, p)}{T_{0}(v, p)}+a_{1}^{2}, \\
& a_{3}=-\frac{T_{3}(v, p)}{T_{0}(v, p)}-a_{1}^{3}+3 a_{1} a_{2}, \\
& a_{4}=-\frac{T_{4}(v, p)}{T_{0}(v, p)}+a_{1}^{4}-6 a_{1}^{2} a_{2}+4 a_{1} a_{3}+3 a_{2}^{2}
\end{aligned}
$$

appearing in the expression for $E_{\mu}$ are presented in terms of special functions

$$
T_{n}(v, p)=\sum_{m=0}^{\infty} \frac{v^{m}}{m !} m^{n} e^{-p m^{2}},
$$

which are rapidly convergent series due to the condition $p>0$. The parameter $p$ depends on temperature and is proportional to the Fourier transform $\Psi(0)$ of the repulsive part of the interaction potential for $k=0$ (see Sec. 2). Thus, the quantities $a_{n}$ are functions of temperature and microscopic parameters of the interaction potential, in particular, of the ratio $R_{0} / \alpha$ characterizing real substances (Morse fluids). Our numerical calculations of the quantities $a_{n}$ are made for $T=T_{c}$. For the coefficients $\gamma_{0 l}$ in the term $P_{a}^{(-)}(T)$ in Eq. (70), we have

$$
\begin{aligned}
\gamma_{01} & =s^{-3} \frac{f_{C R}^{(0)}}{\left(1-s^{-3}\right)}, \\
\gamma_{02} & =s^{-3} \frac{c_{11} d_{1} E_{2}}{1-E_{2} s^{-3}}, \\
\gamma_{03} & =s^{-3}\left[\frac{c_{12} d_{1} E_{2}}{1-E_{2} s^{-3}}+\frac{c_{11}^{2} d_{3} E_{2}^{2}}{1-E_{2}^{2} s^{-3}}\right] .
\end{aligned}
$$

Here

$$
\begin{aligned}
& c_{11}=\beta_{c} W(0) D^{-1}\left(\tilde{a}_{2}+2 R^{(0)} \beta_{c} W(0) a_{4}\left(u^{*}\right)^{-1 / 2}\right), \\
& c_{12}=-\beta_{c} W(0) D^{-1}\left(\tilde{a}_{2}+3 R^{(0)} \beta_{c} W(0) a_{4}\left(u^{*}\right)^{-1 / 2}\right),
\end{aligned}
$$

and $f_{C R}^{(0)}, d_{l}$ are ultimately expressed by the coordinates of a fixed point [14,20]. The quantities $D$ and $R^{(0)}$ are determined by the eigenvalues and 
elements of the renormalization group linear transformation matrix [14]. The form of $\tilde{a}_{2}$ is given in Eqs. (10).

The temperature $k T_{c}$, average density $\bar{n}_{c}$, and pressure $P_{c}$ at the critical point can be calculated using the corresponding equation presented in [14], Eq. (65), and Eq. (70), respectively. Numerical values of $k T_{c}, \bar{n}_{c}$, and $P_{c}$ obtained for $\mathrm{Na}$ and $\mathrm{K}$ from the present researches on the basis of the cell fluid model, from Monte Carlo simulation results for the continuous system with the Morse potential in the grand canonical ensemble [11], and from experiment [26] are given in Table 1. The case of $\mathrm{K}$ corresponds to the set of parameters $R_{0} / \alpha=3.0564$ (see [11]) and $\chi=1.1981(p=2.0100$ ),

Table 1: Numerical estimates of the critical-point parameters $k T_{c}, \bar{n}_{c}$, and $P_{c}$ obtained for sodium and potassium from the present theory, simulations [1], and experiment [26]. Temperature $k T=k T^{\prime} / D$, density $\bar{n}=\rho R_{0}^{3}$, and pressure $P=P^{\prime} R_{0}^{3} / D$ are presented in the form of reduced dimensionless units (the quantities $T^{\prime}, \rho$, and $P^{\prime}$ have dimensional representations, for example, $\left[T^{\prime}\right]=K,[\rho]=1 / m^{3}$, and $\left.\left[P^{\prime}\right]=P a\right)$.

\begin{tabular}{lccccccc}
\hline \multirow{2}{*}{ Research methods } & \multicolumn{4}{c}{$\mathrm{Na}$} & & \multicolumn{3}{c}{$\mathrm{K}$} \\
\cline { 2 - 4 } \cline { 7 - 8 } & $k T_{c}$ & $\bar{n}_{c}$ & $P_{c}$ & & $k T_{c}$ & $\bar{n}_{c}$ & $P_{c}$ \\
\hline Theory (cell fluid model) & 4.028 & 0.997 & 0.474 & & 3.304 & 0.935 & 0.408 \\
Simulations & 5.874 & 1.430 & 2.159 & & 5.050 & 1.125 & 1.651 \\
Experiment & 3.713 & 1.215 & 0.415 & & 3.690 & 0.772 & 0.498 \\
\hline
\end{tabular}

$v=2.9402$ (see [16]). The set of parameters in the case of $\mathrm{Na}$ is given in Sec. 2. The values of coordinates of the critical point for $\mathrm{Na}$, calculated by using the obtained relations, coincide with the corresponding estimates obtained in [14] from an analysis of temperatures above $T_{c}$.

It should be noted that the present approach is aimed at calculating and analyzing the characteristics of the system in a narrow neighborhood of $T_{c}$ $\left(|\tau|<\tau^{*} \sim 10^{-2}\right.$, see [6, 7]), where theoretical and experimental researches are difficult to carry out. The dome of the coexistence curve (the upper portion of the coexistence curve) obtained for $\mathrm{Na}$ in the immediate vicinity of $T_{c}$ on the basis of Eq. (62) at $M=0$ is shown in Fig. 5 (the solid curve). For comparison of the results, the coexistence curves obtained for $\mathrm{Na}$ in the case of the so called zero mode approximation corresponding to the mean-field approximation [16] and from simulation data [11] are plotted in Fig. [5] as well. The zero mode approximation [16] does not take into 


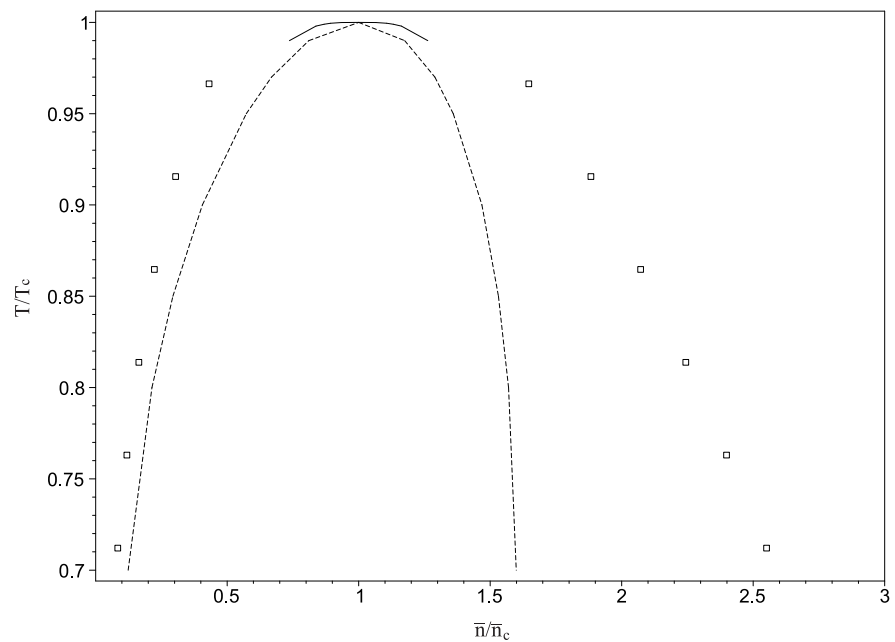

Figure 5: Coexistence curve (binodal curve) for sodium. The solid curve represents the analytic results (the present theory), the dashed curve corresponds to results obtained in the zero mode approximation [16], and boxes represent Monte Carlo simulation results [11].

account the fluctuations of the order parameter. It is not effective in the immediate vicinity of $T_{c}$, where fluctuation effects play a significant role. The approach developed in the present paper for temperatures $T<T_{c}$, takes into account the fluctuation effects that lead to the emergence of a renormalization group symmetry. Fig. 5 clearly demonstrates the deficiency of the zero mode approximation to correctly obtain the branches and dome of the binodal curve near $T_{c}$ (especially the liquid branch). A better quantitative description of the dome is provided by the approach presented here.

The description of the asymmetry of the coexistence curve will be the subject of further studies. It is expected that the asymmetry of the gas and liquid branches of the coexistence curve is associated with the presence of odd powers of the variable in the Jacobian of transition to collective variables. The description of this asymmetry in the proposed approach is a nontrivial problem, whose solution requires a separate analysis and additional calculations. 


\section{Conclusions}

The method for constructing the equation of state of a cell fluid model in the high-temperature region $\left(T>T_{c}\right)$ (see [14]) is generalized to the case of $T<$ $T_{c}$. As in [14, the mathematical description is performed in the vicinity of the critical point by using the collective variables approach with allowance for non-Gaussian fluctuations of the order parameter. The role of the interaction potential is played by the Morse potential possessing the Fourier transform. Calculations of the grand partition function and thermodynamic potential of the model are carried out in the approximation of the quartic fluctuation distribution (the $\rho^{4}$ model). The complete expression for the thermodynamic potential is obtained by summing up the contributions from all regimes of fluctuations in accordance with a calculation technique elaborated in this work for $T<T_{c}$.

For various values of the relative temperature, the variation of the fluid density with increasing chemical potential is traced using the obtained nonlinear equation.

The equation of state obtained in the present research in the case of temperatures below the critical value of $T_{c}$ provides the pressure as a function of temperature and density. The main advantage of this equation of state is the presence of relations connecting its coefficients with the fixed-point coordinates and the microscopic parameters of the interaction potential.

This paper logically supplements the previous study for temperatures above the critical value of $T_{c}$ [14]. As a result of this supplement, an integral critical-behavior picture (the cases of both high- and low-temperature regions are covered) is constructed within the framework of a cell fluid model by using a unified approach.

Although the idea of a microscopic description of the system behavior by the collective variables method at $T<T_{c}$ is similar to that used at $T>T_{c}$ (the separate inclusion of contributions from short-wave and long-wave fluctuations of the order parameter), the temperature region $T<T_{c}$ itself and the results obtained for it differ significantly from the case of $T>T_{c}$ [14]. Let us point out the main differences.

(1) Above the critical temperature $T_{c}$, a liquid cannot exist. Below the critical value of $T_{c}$, two phases can coexist (a gas at low density and a liquid at high density). In contrast to $T>T_{c}$, the system at $T<T_{c}$ acquires a nonzero order parameter (see, for example, Fig. 5, where $\left|\bar{n}-\bar{n}_{c}\right| \neq 0$ for $T<T_{c}$ ). It is not introduced as an independent quantity, but is determined 
as a result of direct calculation. This is possible since the set of collective variables contains the variable $\rho_{0}$ associated with the order parameter.

(2) Due to the different quantities $n_{p}$ and $n_{p}^{\prime}$, which determine the system's points of exit from the critical fluctuation regime at $T>T_{c}$ and $T<T_{c}$ (see Sec. 2), we have the difference in the precise location of the critical regime in each of the temperature cases.

(3) The critical regime of fluctuations is observed above as well as below $T_{c}$. It is characterized by a renormalization group symmetry and is described by a non-Gaussian distribution. In contrast to the limiting Gaussian regime for $T>T_{c}$, the inverse Gaussian regime for $T<T_{c}$ is described by a nonGaussian distribution [unlike the coefficient $r_{n_{p}+2}$ for $T>T_{c}$ (see Fig. 10), the analogous quantity $r_{n_{p}^{\prime}+2}$ appearing in the quadratic coefficient $\bar{g}^{\prime}(k)$ in an exponential function in Eq. (29) takes on negative values for all temperatures $T<T_{c}$ (see Fig. [1a)]. The distribution acquires the Gaussian form [the quantity $\bar{g}^{\prime}(k)$ in Eq. (29) becomes positive] only as a result of the substitution (28).

(4) The contribution $\Omega_{0}^{(-)}$[see Eq. (53) $]$to the total thermodynamic potential is characterized by the coefficients $e_{0}^{(-)}$and $e_{2}^{(-)}$[see Eqs. (44)] and is associated with the shift of the variable $\rho_{0}$. The cubic equation (34) for the quantity $\sigma_{0}^{\prime}$ determining this shift $\sigma_{-}=\sigma_{0}^{\prime} s^{-\left(n_{p}^{\prime}+2\right) / 2}$ is obtained. For $T>T_{c}$, the analogous cubic equation has only one real root [14], which is zero in the absence of the chemical potential. The situation for $\sigma_{0}^{\prime}$ in the temperature region $T<T_{c}$ is more complicated. Unlike the case of $T>T_{c}$, the equation (34) can have more than one real root. The number of real solutions depends on the value of the chemical potential $M$. For all $|M|>M_{q}$ (discriminant $Q>0)$, one real root exists [see Eqs. (37)]. For $|M|<M_{q}(Q<0)$, the cubic equation (34) has three real solutions [see Eqs. (38) and Fig. 2. The magnitude of the chemical potential $M_{q}$ [see Eq. (36)] is found from the condition $Q=0$. It should be noted that the contribution $\Omega_{0}^{(-)}$is nonzero even when $M=0$. For $T>T_{c}$, the analogous contribution $\Omega_{0}^{(+)}[14$ ] is equal to zero in the case when $M=0$.

(5) The function $E_{0}\left(\sigma_{-}\right)$[see Eqs. (30) or (43) and (20) for $s^{-\left(n_{p}^{\prime}+1\right)}$ ] appearing in the contribution $\Omega_{0}^{(-)}=-k T N_{v} E_{0}\left(\sigma_{-}\right)$from the macroscopic part of the variable $\rho_{0}$ coincides in form with the integrand $E_{0}(\rho)$ at the extremum point $\bar{\rho}$, which is the same as $\sigma_{-}$(see Sec. 4). The quantity $\sigma_{0}^{\prime}$ in the expression (33) for $\sigma_{-}$is defined by three real solutions of the cubic equation (34) when the discriminant $Q<0$. From the three real solutions (38), we choose 
one solution that leads to a maximum of $E_{0}\left(\sigma_{-}\right)$. The solution $\sigma_{0}^{\prime}=\sigma_{01}^{\prime}$ corresponds to the maximum of $E_{0}\left(\sigma_{-}\right)$at the chemical potential $M>0$, and the solution $\sigma_{0}^{\prime}=\sigma_{03}^{\prime}$ corresponds to the maximum of $E_{0}\left(\sigma_{-}\right)$at the chemical potential $M<0$. In contrast to $T>T_{c}$, the order parameter distribution characterized by the function $E_{0}\left(\sigma_{-}\right)$exhibits a bimodal shape [27 29].

(6) On the basis of the obtained equation (65), the curves describing the dependence of the density $\bar{n}$ on the chemical potential $M$ are plotted for various values of temperatures $T<T_{c}$ (see Figs. 3 and 4). As is clearly seen from Figs. 3 and 4, a change in sign of $M$ leads to a jump in $\bar{n}$. For $T>T_{c}$, a similar density jump is not observed since the cubic equation has a single solution, which is a continuous function of $M$ (see [14]).

The region in the vicinity of the critical point is interesting (due to the fundamental and applied aspects) and difficult (due to the essential role of fluctuation effects) to analyze. The Morse fluid theory in the vicinity of the critical point is constructed in this work without involving the hard-spheres reference system. The reference system is formed as some part of the repulsive component of the interaction potential in the course of calculating the grand partition function. This allows us to take into account both short-range and long-range interactions from the unified positions of the collective variables approach.

It is expected that the method developed here and in [14 for Morse fluids can be applied to the description of a phase transition in simple liquid alkali metals. Numerical estimates of the critical-point parameters for potassium are obtained in addition to the estimates for sodium. The values of dimesionless critical temperature, density, and pressure, calculated for $\mathrm{Na}$ and $\mathrm{K}$ on the basis of the proposed method, are in accord with the other authors' data. As is seen from Table 1, our values of the critical temperature, density, and pressure agree more closely with experimental data than Monte Carlo simulation results from [11]. In [11], the authors note that the critical pressures for the alkali metals (sodium and potassium) show large deviations from the experimental values. The pressure for $\mathrm{Na}$ is vastly overestimated because the critical temperature is overvalued (see Table 10. It is observed that at the experimental critical point of Na metal, $2485 \mathrm{~K}$, the corresponding pressure predicted by simulation is a good approximation to the experimental critical pressure. Our estimates for the critical temperature $k T_{c}$ in the $\rho^{4}$ model approximation (4.028 for $\mathrm{Na}$ and 3.304 for $\mathrm{K}$, see Table 1) also agree more closely with the experimental values (3.713 for $\mathrm{Na}$ and 3.690 for $\mathrm{K}$, see Table 1) than the corresponding results (5.760 for $\mathrm{Na}$ and 5.037 for K) obtained 
in the previous study [16] in the zero mode approximation (the mean-field approximation). Compared with the previous results (the dashed curve in Fig. (5), our estimates for the binodal dome in the immediate vicinity of $T_{c}$ (the solid curve in Fig. 5) are in better agreement with the predicted data that can be obtained for $\mathrm{Na}$ by extrapolation of the Monte Carlo simulation results [1] (boxes in Fig. [5) to $T / T_{c} \approx 1$.

We hope that the proposed method and explicit representations obtained for a simple fluid system may provide useful benchmarks in studying the critical behavior of a multicomponent fluid. The performed researches also deepen knowledge about the critical properties of fluids and serve as a certain methodological contribution to the theoretical description of critical phenomena.

\section{Appendix A}

\section{Average density components}

Let us write the terms appearing in the expression for $\bar{n}$ [see Eqs. (57) and (58) ].

The first term in Eq. (57)

$$
\bar{n}_{a}=-M-\tilde{a}_{1}+\frac{a_{34}}{\beta W(0)}
$$

corresponds to the analytic part.

The second term in Eq. (57) is represented as

$$
\begin{aligned}
& n_{s}^{(-)}=\left(\tilde{h}^{2}+h_{c m}^{2}\right)^{\frac{d-2}{2(d+2)}}\left[\left(\tilde{h}^{2}+h_{c m}^{2}\right)^{1 / 2} \frac{\partial \gamma_{s}^{(-)}}{\partial \beta \mu}+\frac{2 d}{d+2} \gamma_{s}^{(-)}\right. \\
& \left.\times \frac{1}{(\beta W(0))^{1 / 2}} \frac{\tilde{h}}{\left(\tilde{h}^{2}+h_{c m}^{2}\right)^{1 / 2}}\right] .
\end{aligned}
$$

The third term $n_{0}^{(-)}$in Eq. (57) satisfies the relation

$$
\begin{aligned}
& n_{0}^{(-)}=\left(\tilde{h}^{2}+h_{c m}^{2}\right)^{\frac{d-2}{2(d+2)}}\left[e_{0}^{(-)} \frac{1}{(\beta W(0))^{1 / 2}}\left(1+\frac{d-2}{d+2} \frac{\tilde{h}^{2}}{\tilde{h}^{2}+h_{c m}^{2}}\right)\right. \\
& -\frac{2 d}{d+2} e_{2}^{(-)} \frac{1}{(\beta W(0))^{1 / 2}} \frac{\tilde{h}}{\left(\tilde{h}^{2}+h_{c m}^{2}\right)^{1 / 2}}
\end{aligned}
$$




$$
\left.-\left(\tilde{h}^{2}+h_{c m}^{2}\right)^{1 / 2} \frac{\partial e_{2}^{(-)}}{\partial \beta \mu}\right]
$$

\section{Appendix B}

\section{Quantity $\sigma_{00}^{(-)}$as function of $\alpha_{m}$}

Let us consider the derivatives $\frac{\partial e_{2}^{(-)}}{\partial \beta \mu}$ and $\frac{\partial \gamma_{s}^{(-)}}{\partial \beta \mu}$ appearing in the expression for $e_{02}^{(-)}$[see Eqs. (61), (444), and (다)]. The final formula for $\sigma_{00}^{(-)}$will be given later.

For the derivative of $e_{2}^{(-)}$with respect to $\beta \mu$, we obtain

$$
\frac{\partial e_{2}^{(-)}}{\partial \beta \mu}=-\frac{1}{(\beta W(0))^{1 / 2}} q_{s}^{\prime}\left(\sigma_{0}^{\prime}\right)^{2}\left[1+\frac{q_{l}}{12}\left(\sigma_{0}^{\prime}\right)^{2}\right]\left(\tilde{h}^{2}+h_{c m}^{2}\right)^{-1 / 2} \text {, }
$$

where

$$
\begin{aligned}
& q_{s}^{\prime}=-\frac{E_{2}}{2 p_{0}} q s^{-3} H_{c m} \frac{\alpha_{m}}{\left(1+\alpha_{m}^{2}\right)^{1 / 2}}, \\
& q_{l}=\Phi_{q} u^{*} q^{-1} .
\end{aligned}
$$

Here, it is taken into account that

$$
\begin{aligned}
& \frac{\partial r_{n_{p}^{\prime}+2}}{\partial \beta \mu}=\frac{1}{(\beta W(0))^{1 / 2}} q E_{2} H_{c m d}\left(\tilde{h}^{2}+h_{c m}^{2}\right)^{-1 / 2}, \\
& \frac{\partial u_{n_{p}^{\prime}+2}}{\partial \beta \mu}=\frac{1}{(\beta W(0))^{1 / 2}} u^{*} \Phi_{q} E_{2} H_{c m d}\left(\tilde{h}^{2}+h_{c m}^{2}\right)^{-1 / 2}, \\
& \frac{\partial H_{c m}}{\partial \beta \mu}=\frac{\partial H_{c m}}{\partial \tilde{h}} \frac{\partial \tilde{h}}{\partial \beta \mu}=-\frac{1}{(\beta W(0))^{1 / 2}} H_{c m d}\left(\tilde{h}^{2}+h_{c m}^{2}\right)^{-1 / 2}, \\
& H_{c m d}=\frac{H_{c m}}{p_{0}} \frac{\alpha_{m}}{\left(1+\alpha_{m}^{2}\right)^{1 / 2}} .
\end{aligned}
$$

The derivative of $\gamma_{s}^{(-)}$is calculated on the basis of Eq. (52), where an explicit expression for each term is known. The expression for the derivative

$$
\frac{\partial \gamma_{s}^{(-)}}{\partial \beta \mu}=\frac{\partial f_{n_{p}^{\prime}+1}}{\partial \beta \mu}-\frac{\partial \bar{\gamma}^{(-)}}{\partial \beta \mu}+s^{-3} \frac{\partial f_{I}}{\partial \beta \mu}
$$


is the sum of the derivatives of the quantities $\bar{\gamma}^{(-)}, f_{n_{p}^{\prime}+1}$, and $f_{I} / s^{3}$, which describe contributions from the region of the critical regime of the order parameter fluctuations, the transition region, and the region of the inverse Gaussian regime, respectively.

Let us calculate the derivative of the quantity $f_{n_{p}^{\prime}+1}$ [see Eq. (19) for $\left.n=n_{p}^{\prime}+1\right]$ with respect to the chemical potential. Taking into account the relations

$$
\begin{aligned}
& \frac{\partial y_{n_{p}^{\prime}+m}}{\partial \beta \mu}=y_{n_{p}^{\prime}+m} r_{p+m}^{\prime} \frac{\partial x_{n_{p}^{\prime}+m}}{\partial \beta \mu} \\
& \frac{\partial x_{n_{p}^{\prime}+m}}{\partial \beta \mu}=\frac{1}{(\beta W(0))^{1 / 2}} g_{p+m}^{\prime}\left(\tilde{h}^{2}+h_{c m}^{2}\right)^{-1 / 2}, \\
& U^{\prime}\left(0, x_{n_{p}^{\prime}+m}\right)=-\frac{x_{n_{p}^{\prime}+m}}{2} U\left(0, x_{n_{p}^{\prime}+m}\right)-\frac{1}{2} U\left(1, x_{n_{p}+m}^{\prime}\right),
\end{aligned}
$$

where

$$
\begin{aligned}
& r_{p+m}^{\prime}=\frac{U^{\prime}\left(x_{n_{p}^{\prime}+m}\right)}{U\left(x_{n_{p}^{\prime}+m}\right)}-\frac{1}{2} \frac{\varphi^{\prime}\left(x_{n_{p}^{\prime}+m}\right)}{\varphi\left(x_{n_{p}^{\prime}+m}\right)}, \\
& g_{p+m}^{\prime}=\bar{x} H_{c m d} E_{2}^{m-1}\left(1-\Phi_{q} H_{c m} E_{2}^{m-1}\right)^{-1 / 2} \\
& \times\left[1+\frac{\Phi_{q}}{2} H_{c m} E_{2}^{m-1}\left(1-\Phi_{q} H_{c m} E_{2}^{m-1}\right)^{-1}\right], \\
& U^{\prime}\left(x_{n_{p}^{\prime}+m}\right)=\frac{1}{2} U^{2}\left(x_{n_{p}^{\prime}+m}\right)+x_{n_{p}^{\prime}+m} U\left(x_{n_{p}^{\prime}+m}\right)-1, \\
& \varphi^{\prime}\left(x_{n_{p}^{\prime}+m}\right)=6 U^{\prime}\left(x_{n_{p}^{\prime}+m}\right) U\left(x_{n_{p}^{\prime}+m}\right)+2 U\left(x_{n_{p}^{\prime}+m}\right) \\
& +2 x_{n_{p}^{\prime}+m} U^{\prime}\left(x_{n_{p}^{\prime}+m}\right),
\end{aligned}
$$

we find

$$
\frac{\partial f_{n_{p}^{\prime}+1}}{\partial \beta \mu}=\frac{1}{(\beta W(0))^{1 / 2}} f_{p}^{\prime}\left(\tilde{h}^{2}+h_{c m}^{2}\right)^{-1 / 2}
$$

Here

$$
f_{p}^{\prime}=\frac{1}{2} r_{p}^{\prime} g_{p}^{\prime}\left(1-9 / y_{n_{p}^{\prime}}^{2}\right)-\frac{1}{2} g_{p+1}^{\prime} U\left(x_{n_{p}^{\prime}+1}\right) .
$$

The derivative of the quantity $\bar{\gamma}^{(-)}$[see Eq. (22)] with respect to the chemical potential is written in the form

$$
\frac{\partial \gamma^{(-)}}{\partial \beta \mu}=-\frac{1}{(\beta W(0))^{1 / 2}} \gamma_{p}^{\prime}\left(\tilde{h}^{2}+h_{c m}^{2}\right)^{-1 / 2},
$$


where

$$
\gamma_{p}^{\prime}=H_{c m d}\left(-\bar{\gamma}_{2}+2 \bar{\gamma}_{3} H_{c m}\right)
$$

The calculation of the derivative of $f_{I}$ [see Eq. (47)] with respect to $\beta \mu$ will be carried out taking into account that the quantity $\sigma_{0}^{\prime}$ is a function of the chemical potential. Using the equality (32), we obtain the relation

$$
\frac{\partial \sigma_{0}^{\prime}}{\partial \beta \mu}=\frac{1}{(\beta W(0))^{1 / 2}} g_{\sigma}^{\prime}\left(\tilde{h}^{2}+h_{c m}^{2}\right)^{-1 / 2},
$$

which makes it possible to find the following expression for the derivative of the quantity $r_{R}^{\prime}$ appearing in Eq. (47):

$$
\frac{\partial r_{R}^{\prime}}{\partial \beta \mu}=\frac{1}{(\beta W(0))^{1 / 2}} g_{R}^{\prime}\left(\tilde{h}^{2}+h_{c m}^{2}\right)^{-1 / 2} .
$$

Here

$$
\begin{aligned}
& g_{\sigma}^{\prime}=\frac{s^{5 / 2}}{r_{R}^{\prime}} \frac{1}{1+\alpha_{m}^{2}}-\frac{\sigma_{0}^{\prime}}{r_{R}^{\prime}} H_{c m d} q E_{2}\left(1+\frac{q_{l}}{6}\left(\sigma_{0}^{\prime}\right)^{2}\right) \\
& g_{R}^{\prime}=q E_{2} H_{c m d}\left(1+\frac{q_{l}}{2}\left(\sigma_{0}^{\prime}\right)^{2}\right)+u_{n_{p}^{\prime}+2} g_{\sigma}^{\prime} \sigma_{0}^{\prime} .
\end{aligned}
$$

Another obtained formula

$$
\frac{\partial a_{I}}{\partial \beta \mu}=\frac{1}{(\beta W(0))^{1 / 2}} g_{a I}\left(\tilde{h}^{2}+h_{c m}^{2}\right)^{-1 / 2},
$$

where

$$
g_{a I}=-\frac{a_{I} g_{R}^{\prime}}{2 r_{R}^{\prime}}
$$

allows us to calculate the derivative of the quantity $f_{I}^{\prime \prime}$ [see Eqs. (48)] appearing in the expression (47) for $f_{I}$. We have

$$
\frac{\partial f_{I}^{\prime \prime}}{\partial \beta \mu}=\frac{1}{(\beta W(0))^{1 / 2}} g_{a I} a_{I g}\left(\tilde{h}^{2}+h_{c m}^{2}\right)^{-1 / 2} .
$$

Here

$$
a_{I g}=\frac{2 a_{I}}{1+a_{I}^{2}}-\frac{4}{a_{I}^{3}}+\frac{6}{a_{I}^{4}} \arctan a_{I}-\frac{2}{a_{I}^{3}} \frac{1}{1+a_{I}^{2}} .
$$

Using Eqs. (B.5) and (B.6), we arrive at the expression

$$
\frac{\partial f_{I}}{\partial \beta \mu}=\frac{1}{(\beta W(0))^{1 / 2}} f_{I v}\left(\tilde{h}^{2}+h_{c m}^{2}\right)^{-1 / 2},
$$


where

$$
\begin{aligned}
& f_{I v}=\frac{1}{4} \frac{u^{*} \Phi_{q}}{u_{n_{p}^{\prime}+1}} H_{c m d}-\frac{1}{2}\left(\frac{g_{R}^{\prime}}{r_{R}^{\prime}}+g_{a I} a_{I g}\right) \\
& +g_{p+1}^{\prime}\left(\frac{3}{4} \frac{r_{p+1}^{\prime}}{y_{n_{p}^{\prime}+1}^{2}}-\frac{1}{2} \frac{U^{\prime}\left(x_{n_{p}^{\prime}+1}\right)}{U\left(x_{n_{p}^{\prime}+1}\right)}\right) .
\end{aligned}
$$

With allowance for Eqs. (B.3), (B.4), and (B.7), the derivative from Eq. (B.2) can be represented as

$$
\frac{\partial \gamma_{s}^{(-)}}{\partial \beta \mu}=\frac{1}{(\beta W(0))^{1 / 2}} f_{\delta_{1}}\left(\tilde{h}^{2}+h_{c m}^{2}\right)^{-1 / 2} .
$$

Here

$$
f_{\delta_{1}}=f_{p}^{\prime}+\gamma_{p}^{\prime}+f_{I v} / s^{3} .
$$

The quantities $f_{p}^{\prime}, \gamma_{p}^{\prime}$, and $f_{I v}$ depend only on the parameter $\alpha_{m}$.

The above expressions (61), (B.1), and (B.8) make it possible to write the coefficient $\sigma_{00}^{(-)}$, Eq. (60), in the final explicit form. We have

$$
\begin{aligned}
& \sigma_{00}^{(-)}=e_{0}^{(-)} \frac{1}{(\beta W(0))^{1 / 2}}\left(1+\frac{d-2}{d+2} \frac{\alpha_{m}^{2}}{1+\alpha_{m}^{2}}\right) \\
& +e_{00}^{(-)} \frac{\alpha_{m}}{\left(1+\alpha_{m}^{2}\right)^{1 / 2}}+e_{02}^{(-)}
\end{aligned}
$$

where

$$
e_{02}^{(-)}=\frac{1}{(\beta W(0))^{1 / 2}}\left[f_{\delta_{1}}+q_{s}^{\prime}\left(\sigma_{0}^{\prime}\right)^{2}\left(1+q_{l}\left(\sigma_{0}^{\prime}\right)^{2} / 12\right)\right] .
$$

Thus, the quantity $\sigma_{00}^{(-)}$is a function of

$$
\alpha_{m}=\frac{\tilde{h}}{h_{c m}}=(\beta W(0))^{1 / 2}\left(\frac{q}{c_{11}} E_{2}^{-n_{0}}\right)^{p_{0}} \alpha_{0} .
$$

The multiplier

$$
\alpha_{0}=\frac{M}{|\tau|^{p_{0}}}=\frac{\mu / W(0)-\tilde{a}_{1}}{|\tau|^{p_{0}}}
$$

includes the initial quantities $\mu$ and $\tau$. 


\section{References}

[1] J.-P. Hansen, I. R. McDonald, Theory of Simple Liquids: With Applications to Soft Matter, Academic Press, Oxford, 2013.

[2] K. N. Khanna, I. L. McLaughlin, J. Phys.: Condens. Matter 1 (1989) 4155 .

[3] D. Ben-Amotz, G. Stell, J. Phys. Chem. B 108 (2004) 6877.

[4] R. Roth, J. Phys.: Condens. Matter 22 (2010) 063102.

[5] I. R. Yukhnovskii, Condens. Matter Phys. 17 (2014) 43001.

[6] I. R. Yukhnovskii, Phase Transitions of the Second Order. Collective Variables Method, World Scientific, Singapore, 1987.

[7] I. R. Yukhnovskii, M. P. Kozlovskii, I. V. Pylyuk, Microscopic Theory of Phase Transitions in the Three-Dimensional Systems, Eurosvit, Lviv, 2001 [in Ukrainian].

[8] A. L. Rebenko, Rev. Math. Phys. 25 (2013) 1330006.

[9] V. A. Boluh, A. L. Rebenko, J. Mod. Phys. 6 (2015) 168.

[10] H. Okumura, F. Yonezawa, J. Chem. Phys. 113 (2000) 9162.

[11] J. K. Singh, J. Adhikari, S. K. Kwak, Fluid Phase Equilib. 248 (2006) 1 .

[12] E. M. Apfelbaum, J. Chem. Phys. 134 (2011) 194506.

[13] A. Martínez-Valencia, M. González-Melchor, P. Orea, J. López-Lemus, Mol. Simul. 39 (2013) 64.

[14] M. P. Kozlovskii, I. V. Pylyuk, O. A. Dobush, Condens. Matter Phys. 21 (2018) 43502.

[15] I. R. Yukhnovskii, M. P. Kozlovskii, I. V. Pylyuk, Phys. Rev. B 66 (2002) 134410.

[16] M. P. Kozlovskii, O. A. Dobush, I. V. Pylyuk, Ukr. J. Phys. 62 (2017) 865. 
[17] M. P. Kozlovskii, I. V. Pylyuk, I. R. Yukhnovskii, Theor. Math. Phys. 87 (1991) 540.

[18] M. P. Kozlovskii, R. V. Romanik, J. Phys. Stud. 13 (2009) 4007.

[19] J. Engels, L. Fromme, M. Seniuch, Nucl. Phys. B 655 (2003) 277.

[20] M. P. Kozlovskii, The Influence of an External Field on the Critical Behavior of the Three-Dimensional Systems, Galytskii Drukar, Lviv, 2012 [in Ukrainian].

[21] Handbook of Mathematical Functions with Formulas, Graphs and Mathematical Tables, edited by Milton Abramowitz, Irene A. Stegun, National Bureau of Standards, Applied Mathematics Series 55, 1964.

[22] M. P. Kozlovskii, I. V. Pylyuk, I. R. Yukhnovskii, Theor. Math. Phys. 87 (1991) 641.

[23] M. P. Kozlovskii, I. V. Pylyuk, O. O. Prytula, Phys. Rev. B 73 (2006) 174406 .

[24] M. P. Kozlovskii, I. V. Pylyuk, O. O. Prytula, Nucl. Phys. B 753 (2006) 242.

[25] I. V. Pylyuk, J. Magn. Magn. Mater. 305 (2006) 216.

[26] F. Hensel, J. Phys.: Condens. Matter 2 (1990) SA33.

[27] K. Binder, D. P. Landau, Phys. Rev. B 30 (1984) 1477.

[28] K.-C. Lee, Phys. Rev. E 53 (1996) 6558.

[29] Ph. Chomaz, F. Gulminelli, Physica A 330 (2003) 451. 